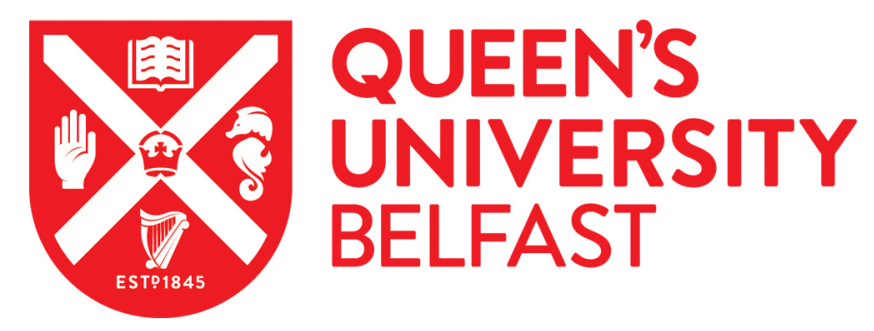

\title{
A New Optimal Sliding Mode Controller with Adjustable Gains based on Bolza-Meyer Criterion for Vibration Control
}

Do, X. P., Mien, V., Thanh Tu, P. H., Nguyen, N. P., \& Choi, S-B. (2020). A New Optimal Sliding Mode Controller with Adjustable Gains based on Bolza-Meyer Criterion for Vibration Control. Journal of Sound and Vibration, 485, [115542]. https://doi.org/10.1016/j.jsv.2020.115542

Published in:

Journal of Sound and Vibration

Document Version:

Peer reviewed version

Queen's University Belfast - Research Portal:

Link to publication record in Queen's University Belfast Research Portal

\section{Publisher rights}

Copyright 2020 Elsevier.

This manuscript is distributed under a Creative Commons Attribution-NonCommercial-NoDerivs License

(https://creativecommons.org/licenses/by-nc-nd/4.0/), which permits distribution and reproduction for non-commercial purposes, provided the author and source are cited.

\section{General rights}

Copyright for the publications made accessible via the Queen's University Belfast Research Portal is retained by the author(s) and / or other copyright owners and it is a condition of accessing these publications that users recognise and abide by the legal requirements associated with these rights.

Take down policy

The Research Portal is Queen's institutional repository that provides access to Queen's research output. Every effort has been made to ensure that content in the Research Portal does not infringe any person's rights, or applicable UK laws. If you discover content in the Research Portal that you believe breaches copyright or violates any law, please contact openaccess@qub.ac.uk. 


\title{
A New Optimal Sliding Mode Controller with Adjustable Gains based on Bolza-Meyer Criterion for Vibration Control
}

\author{
Do Xuan Phu ${ }^{1 *}$, Van Mien ${ }^{2}$, Phan Huu Thanh Tu ${ }^{3}$, Ngoc Phi Nguyen ${ }^{4}$, Seung-Bok Choi ${ }^{* *}$ \\ ${ }^{1}$ Department of Mechatronics and Sensor Systems Technology, Vietnamese-German University, Binh \\ Duong, Vietnam, Email: phu.dx@vgu.edu.vn \\ ${ }^{2}$ School of Electronics, Electrical Engineering and Computer Science, Queen's University Belfast, \\ BT71NN, UK. Email: m.van@qub.ac.uk \\ ${ }^{3}$ Department of Mechatronics and Sensor Systems Technology, Vietnamese-German University, Binh \\ Duong, Vietnam. Email: thanhtuphanhuи@gmail.com \\ ${ }^{4}$ Department of Aerospace Engineering, Sejong University, Seoul, Korea. Email: npnguyen@sejong.ac.kr \\ ${ }^{5}$ Smart Structures and Systems Laboratory, Department of Mechanical Engineering, Inha University, \\ Incheon 22212, Korea. Email: seungbok@inha.ac.kr
}

*Corresponding Author: Prof. Seung-Bok Choi (Email:seungbok@inha.ac.kr) and Dr. Do Xuan Phu (Email:phu.dx@vgu.edu.vn) 


\begin{abstract}
In this study, a new optimal control law associated with sliding mode control is developed based on basis of the Bolza-Meyer criterion. The salient characteristic of the proposed controller is to have gains adjustability, where the gain values can be larger than one. This leads to enhancing control system performances with a given cost function. It should be pointed out that conventional optimal controllers usually have constant gains of one or less than one, and hence the control system performances such as the requirement on convergence speed may not be satisfactory. After formulating the proposed optimal control law for polynomial time-varying control systems, computer simulations are carried out to validate the benefits of the proposed approach. Firstly, as an illustrative example, three crucial index values including control gain index, main input control index and the state index are investigated. Secondly, the proposed controller is applied to a vehicle seat suspension system with magneto-rheological damper to evaluate vibration control performances. In simulation studies, a comparison between the proposed approach and a state-of-the-art optimal controller is undertaken to demonstrate additional benefits such as less power and faster convergence of the proposed optimal controller.
\end{abstract}

Keywords: Optimal control, optimal sliding mode control, gain adjustability, Bolza-Meyer criterion, faster convergence, cost function, Hamiltonian function

\title{
1. Introduction
}

In the design of optimal controllers, Hamiltonian function is frequently used to formulate the governing equations of state and co-state models, which are usually required in deriving controllers' formulations. Recently, many research works on the development of optimal controllers have been undertaken to improve control performances in terms of less cost function and higher stability. In addition, several optimal controllers, which are robust against the system uncertainties and disturbances, have been proposed by many researchers. For instance, an adaptive optimal control for unknown constrained input systems has been developed in [1], where Hamilton-Jacobi-Bellman has been used. The result is then combined with a neural network for dealing with the uncertain parameters of the system. Another study of adaptive optimal control has been presented in [2], where a modification of Hamiltonian function has been applied, namely Hamilton-Jacobi-Isaacs function. An integration of Hamiltonian function and Riccati-like equation has also been studied in [3]. Consequently, an adaptive optimal controller for the decentralized system was then formulated. It is well-known that the Hamilton-Jacobi-Bellman has been successfully utilized for optimal control of unknown systems in the presence of disturbances [4], actuator saturation [5], and uncertain dynamics [6]. An optimal control based on only Bellman function has been presented in [7], in which the 
control model is a combination between the conventional function of Bellman and the term of Meyer model. An optimal control law based on only Riccati-like equation to deal with unknown continuous time linear systems has been introduced in [8]. On the other hand, the Bolza-Meyer criterion associated with the Hamiltonian function was also frequently utilized to formulate advanced optimal control laws [9-13]. In these studies [9-13], the Hamiltonian function was used as a basis formulation to derive new optimal controllers. In addition, the application of sign function in the sliding mode control was undertaken to design new advanced optimal control laws, which can produce several significant merits such as less control effort and higher stability. In the development of optimal sliding mode controllers, the adaptive gain approach for linear systems has been adopted and combined with the sliding mode control to increase the robustness of the controller against the parameter uncertainties [14]. In recent years, several optimal sliding mode controllers based on Riccati-like equation, where the sliding mode controllers and fuzzy neural network approximations are integrated, have been proposed [15-18]. In these studies, the modified Riccatilike equation was embedded parameters of fuzzy model/or fuzzy neural model to improve performance of the system. In addition, the parameters of the Riccati equation was also designed as an adaptative law to increase the system under the effects of uncertainty/or severe disturbances. Recently, some modifications of the sliding mode control have been used in adaptive control and double-layer, as given in [20-22]. In these studies, the sliding surface was designed as PID-like type combined with the classical type using sign function to improving performance of the controller. The objective of this combination was to use the advantage of the PID model in tracking error and the capacity in tackling the variation of disturbance of sign function in the sliding mode control.

From the above literature survey, many types of optimal control laws have been developed to enhance performances and robustness of the systems, which are subjected to unknown parameters and disturbances. However, development of new optimal control laws, which can provide better control performances compared to the existing optimal control laws, is still necessary and important. In fact, the design of optimal controllers based on critical criteria such as Meyer, Bolza-Meyer, Hamiltion-Jacobi-Bellman, and Hamiltion-Jacobi-Isaasc term needs to be improved because the value of definite symmetry matrices (gains) is one or less than one, which reduces both control performances and robustness of the optimal controllers [9-13]. This issue becomes even more serious when the conventional models of the optimal control laws are applied into practical engineering applications.

Motivated by the aforementioned issues, the major contributions of this work are twofold. First, a new optimal control law, which can eliminate the drawbacks of the conventional optimal controllers, is developed based on the certain criterion used in [9-13]. Second, a new optimal sliding mode controller based on the basis of the Bolza-Meyer criterion is formulated. Technically, the innovative features of the proposed approach can be pointed out as below. 
i) The Meyer term in the Hamilton-Jacobi-Bellman is designed with a chosen constant to reduce control energy.

ii) The critical issue of definite symmetry matrices of the Hamilton-Jacobi-Bellman with values larger than one is thoroughly solved to provide both high control performances and robustness.

iii) The Bolza-Meyer method is used along with a modification of the Meyer term to simplify the optimal control structure.

These technical contributions are theoretically proved and verified based on the simulation results. In the simulations, in order to demonstrate the advantages of the proposed controller, it is compared with a representative conventional optimal controller. After demonstrating the merit features of the proposed approach through an illustrative example, it is then applied to a vehicle seat suspension system to demonstrate the effectiveness of the proposed approach in terms of suppressing the vibration of the system under the random road excitation.

\section{Design of Optimal Control Law}

A polynomial time-varying system with linear control input is considered as follows [11]:

$\dot{x}(t)=f_{\xi}(x, t)+B_{\xi}(t) u(t)$

where, $x(t) \in R^{n}$ is the state vector, $u(t) \in R^{l}$ is the control input, $B_{\xi}(t)$ is the continuous function with respect to time domain. The system (1) is assumed to be controllable. $f_{\xi}(x, t)$ is an $n$ variables polynomial function with time-dependent continuous coefficients defined as follows:

$f_{\xi}(x, t)=a_{0 \xi}(t)+a_{1 \xi}(t) x(t)+a_{2 \xi}(t) x(t) x^{T}+\ldots+a_{p \xi}(t) x(t) \ldots p$ times $\ldots x(t)$.

In the above equation, $a_{1 \xi}$ is the vector of dimension $n, a_{1 \xi}$ is the matrix of dimension $n \times n, a_{p \xi}$ is the $(p+1)$ dimension tensor of dimension $n \times \ldots(p+1)$ times $\cdots \times n$, and $x \times \ldots$, times $\cdots \times x$ is the $p$-dimension tensor of dimension $n \times \ldots p$ times $\ldots \times n$ obtained by $p$ times spatial multiplication of the vector $x(t)$ by itself. In the classical optimal control problem, the criterion to be minimized is determined by Bolza function as follows:

$$
J_{1}=\frac{1}{2}[x(T)]^{T} \psi_{\xi}[x(T)]+\frac{1}{2} \int_{t o}^{T}\left(u^{T}(s) R_{\xi}(s) u(s)+x^{T}(s) L_{\xi}(s) x(s)\right) d s
$$

where, $R_{\xi}(t)$ is the positive definite symmetric matrix function, $\psi_{\xi}, L_{\xi}(t)$ are symmetric-positive-define matrices; $\psi_{\xi} \geq 0, L_{\xi}(t) \geq 0$. From Eq. (2), the cost function is proposed as follows: 


$$
J_{2}=\sum_{i=1}^{n} \frac{\psi_{\xi i i}\left|x_{i}(T)\right|}{\alpha}+\frac{1}{2} \int_{t o}^{T}\left(u^{T}(s) R_{\xi}(s) u(s)+x^{T}(s) L_{\xi}(s) x(s)\right) d s
$$

where, $\alpha$ is the positive constant of the Meyer part of the cost function. Then, the optimal control law for the system (1) is determined as follows:

$u^{*}(t)=-R_{\xi}^{-1}(t) B_{\xi}{ }^{T}(t) q(t)$

where, $q(t)$ is defined with the signum (sign) function as follows:

$q(t)=\alpha Q_{\xi}(t) \operatorname{sign}[x(t)]$

where, $x=\left[x_{1}, \ldots, x_{n}\right] \in R^{n}, \operatorname{sign}[x(t)]=\left[\operatorname{sign}\left(x_{1}\right), \ldots, \operatorname{sign}\left(x_{n}\right)\right] \in R^{n} . Q_{\xi}(t)$ is the square symmetric matrix $(n \times n)$. From Eq. (5), Eq. (4) can be rewritten as follows:

$u^{*}(t)=-R_{\xi}^{-1}(t) B_{\xi}{ }^{T}(t)\left(\alpha Q_{\xi}(t) \operatorname{sign}[x(t)]\right)$

In the above equation, $Q_{\xi}(t)$ is the square symmetric matrix $(n \times n)$. This matrix is called gain matrix of the system.

Substituting Eq. (6) into Eq. (1), the system (1) is rewritten as follows:

$\dot{x}(t)=f_{\xi}(x, t)-B_{\xi}(t) R_{\xi}^{-1}(t) B_{\xi}{ }^{T}(t)\left(\alpha Q_{\xi}(t) \operatorname{sign}[x(t)]\right)$

From Eq. (6), the new matrix function $Q_{\Delta}(t)$ is defined by

$\dot{Q}_{\Delta}(t)=L_{\xi} x(t)^{2}-\alpha x(t) Q_{\xi}(t)$

where, $Q_{\Delta}=\alpha Q_{\xi}(t)|x(t)|$. Substituting Eq. (8) into Eq. (6), the control $u^{*}(t)$ with new value $Q_{\xi}{ }^{*}(t)$ is derived as follows:

$u^{*}(t)=-R_{\Sigma}^{-1}(t) B_{\Sigma}^{T}(t) Q_{\xi}^{*}(t) \operatorname{sign}[x(t)]$

where, $Q_{\xi}{ }^{*}(t)=\alpha Q_{\Delta}(t)$. The gain matrix function $Q_{\Delta}(t)$ can be extracted from the following derivative function:

$\dot{Q}_{\Delta}(t)=-L_{\xi}(t)|x(t)|-\alpha\left[a_{1 \xi}(t)+2 a_{2 \xi}(t) x(t)+3 a_{3 \xi}(t) x(t) x^{T}(t)+\cdots+p a_{p \xi}(t) x(t) \cdots_{p-1 \text { times }}, \cdots x(t)\right]^{T} Q_{\Delta}(t)|x(t)|$

The detailed derivation of the above equation is presented in Appendix. Consequently, Eq. (7) can rewritten as follows:

$\dot{x}(t)=f_{\xi}(x, t)-B_{\xi}(t) R_{\xi}^{-1}(t) B_{\xi}{ }^{T}(t)\left(\alpha Q_{\Delta}(t) \operatorname{sign}[x(t)]\right)$ 
From Eq.(10), there are some important remarks that can be used to improve performance of the system. These remarks are different from the results reported in reference [11].

Remark 1. In [11], the gain matrix function (10) was obtained as:

$\dot{Q}_{\Delta}(t)=L_{\xi}(t)|x(t)|-\left[a_{1 \xi}(t)+2 a_{2 \xi}(t) x(t)+3 a_{3 \xi}(t) x(t) x^{T}(t)+\cdots+p a_{p \xi}(t) x(t) \cdots_{p-1 \text { times },} \cdots x(t)\right]^{T} Q_{\Delta}(t)(12)$

The result in [11] has a limitation in terms of instability problem of the system. It is not able to use the signum function to derive the gain matrix function, as shown in Eq. (12). This reason is arisen from the standard property of the signum function that the output is saturated between the upper and lower values; 1 and -1. Therefore, the applications of Eq. (12) in real systems are limited in finding the optimal values for the controllers. The subsequent simulation section will show this disadvantage of the conventional approaches.

Remark 2. In [11], the final gain matrix value was defined before proceeding to define the control formulation. This is not an advantage. In conventional optimal controllers, the convergence time of the control can be predicted. However, this property cannot be applied for estimating the gain value. Therefore, the shooting method was utilized for predicting the gain value in [11]. As has been discussed in the literature, the shooting method is an unreliable method and cannot be used for computing when the controller is applied for real systems. In this case, the $4^{\text {th }}$ order Runge-Kutta method should be used instead of the shooting method. From this point of view, the methods used in [11] are not appropriate for achieving high control performances as well as enhancing system robustness.

The gain matrix function $Q_{\Delta}(t)$, as shown in Eq. (10), is not sufficient for controlling system because the negative energy in the right part would decrease the required energy of the control system. Hence, the maximum boundary based on the elements of the right part is suggested as follows:

$\dot{Q}_{\Delta}(t)=L_{\xi}(t) x^{2}(t)-\alpha x(t) Q_{\Delta}(t)$

Eq. (13) is the same as Eq. (8) shown in the above analysis. In this proposed equation (13), the squared value of $x(t)$ gives a larger gain value than that of using the absolute value. This observation will be demonstrated in the simulation section.

Remark 3. It is remarked that the values of $R_{\xi}(t), L_{\xi}(t)$ are defined as a real symmetric positive semidefinite matrix and a real symmetric positive definite matrix, respectively. These matrices represent the relative importance of quadratic terms in the various states $\left(L_{\xi}(t)\right)$ and quadratic term in the control inputs 
$\left(R_{\xi}(t)\right)$ to the overall quadratic objective function (3). In [11], the gain matrix function was designed such that the value of matrices $R_{\xi}(t), L_{\xi}(t)$ were one for any cases. The objective of this choice is to compromise the errors in the quadratic terms. In addition, this choice also supports for minimizing the quadratic terms in the state of the system so that the use of control input will not affect to the convergence of the minimization progress. This is also a disadvantage of the controller in [11] in solving the control problem for the system with different values of $R_{\xi}(t), L_{\xi}(t)$. Hence, in the proposed method of this study, there are no boundaries for the values of $R_{\xi}(t), L_{\xi}(t)$ with the new gain matrix function $Q_{\Delta}(t)$.

Remark 4. The appearance of $\alpha$ in the proposed method is used for adjusting the energy of the main input control $u$ and the energy in the gain matrix function. This modification will optimize the computing progress as well as improving performance of the system. The value $\alpha$ is positive and is selected by trialand-error method.

Remark 5. From Eq. (13), another new equation of the gain matrix function can be used as follows: $\dot{Q}_{\Delta}(t)=L_{\xi}(t)|x(t)|-\alpha\left[a_{1 \xi}(t)+2 a_{2 \xi}(t) x(t)+3 a_{3 \xi}(t) x(t) x^{T}(t)+\cdots+p a_{p \xi}(t) x(t){ }{ }_{p-1 \text { times },} \cdots x(t)\right]^{T} Q_{\Delta}(t)|x(t)|$

However, this equation does not provide good performance compared to Eq. (13). As explained in Remark 3, the matrices $R_{\xi}(t), L_{\xi}(t)$ are not unit matrices, and the quadratic errors related the quadratic terms are also different. Hence, the first order of absolute variable $|x(t)|$ in terms of $L_{\xi}(t)|x(t)|$ are not sufficient for controlling the system because the second element of (14) is negative. This will be demonstrated in the simulation section.

Theorem. If the control system (1) with the cost function (3) is integrated with the control law (9) and the derivative of the gain matrix function (13), then, the optimal control state of the nonlinear system (1) can be rewritten by the system (11).

Proof. The Hamiltonian function candidate of optimal control is established by

$$
H(x, u, q, t)=\frac{1}{2}\left(u^{T} R_{\xi}(t) u+x^{T} L_{\xi}(t) x\right)+q^{T} \dot{x}(t)
$$

The optimal control law $u$ is then extracted by using maximum principle $\frac{\partial H}{\partial u}=0$ as follows: 
$u^{*}(t)=-R_{\xi}^{-1}(t) B_{\xi}^{T}(t) q(t)$

Substituting Eq. (5) into Eq. (16), the main control input is obtained:

$u^{*}(t)=R_{\xi}^{-1}(t) B_{\xi}^{T}(t)\left(\alpha Q_{\xi}(t) \operatorname{sign}[x(t)]\right)$

Applying the co-state equation given by $\frac{d q(t)}{d t}=-\frac{\partial H}{\partial x}$, the following equation is obtained:

$-\frac{d q(t)}{d t}=L_{\xi}(t) x(t)+\left[\frac{\partial f_{\xi}(x, t)}{\partial x}\right]^{T} q(t)$

From Eq. (5) and the definition of $f_{\xi}(x, t)$, Eq. (18) can be rewritten as follows:

$-\alpha \dot{Q}_{\xi}(t) \operatorname{sign}[x(t)]-\alpha Q_{\xi}(t) \frac{d(\operatorname{sign}[x(t)])}{d t}=L_{\xi}(t) x(t)+\alpha\left[\frac{\partial f_{\xi}(x, t)}{\partial x}\right]^{T} Q_{\xi}(t) \operatorname{sign}[x(t)]$

At this stage, the derivative of $f_{\xi}(x, t)$ can be obtained by

$\frac{\partial f_{\xi}(x, t)}{\partial x}=a_{1 \xi}(t)+2 a_{2 \xi}(t) x+3 a_{3 \xi}(t) x x^{T}+\ldots+p a_{p \xi}(t) x(t) \ldots(p-1)$ times $\ldots x(t)$

Using Eqs. (19) and (20), the following equation is obtained:

$$
\begin{aligned}
& -\alpha \dot{Q}_{\xi}(t) \operatorname{sign}[x(t)]-\alpha Q_{\xi}(t) \frac{d(\operatorname{sign}[x(t)])}{d t}=L_{\xi}(t) x(t)+
\end{aligned}
$$

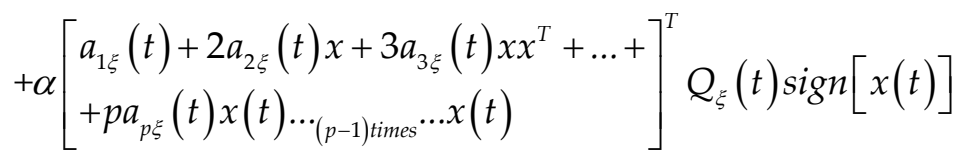

Using the property of $\frac{d(\operatorname{sign}[x(t)])}{d t}=0$, Eq. (21) is re-expressed as follows:

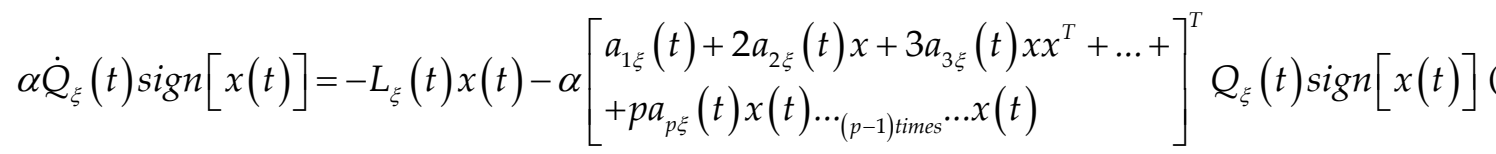

Thus, if $x(t)=0$, then $u(t)=0$, and the value of $Q_{\xi}(t)$ is not required. If $Q_{\xi}(t)$ is the solution of Eq. (8), the result in Eq. (10) is satisfactory. On the other hand, Eq. (5) can be rewritten at time $t=T$ as follows:

$$
q(T)=\alpha Q_{\xi}(T) \operatorname{sign}[x(T)]=\frac{\partial J}{\partial x(T)}=\frac{\psi_{\xi}}{\alpha} \operatorname{sign}[x(T)]
$$

Hence, the value $Q_{\xi}(T)$ can be found from the following equation. 
$Q_{\xi}(T)=\frac{\psi_{\xi}}{\alpha^{2}}$

This result shows that the value $Q_{\xi}(t)$ is a mean value, and the control input $u(t)$ is not zero. This result shows that the optimal control input is continuously calculated to optimize the responses of the system. As mentioned above, if $x(t)=0$, then $u(t)=0$, and the value of $Q_{\xi}(t)$ is not required, the response of the system in this case is stabilized around zero. This completes the proof.

Remark 6. The value $\frac{\psi_{\xi}}{\alpha^{2}}$ of Eq. (24) shows that the final value of $Q_{\xi}(T)$ is a positive value. This is different from the result in [11], which showed a zero value at the time T. The value $\frac{\psi_{\xi}}{\alpha^{2}}$ can be seen as a reference value for the optimal control system.

Remark 7. In the proposed controller, the sliding mode control is applied as shown in (4) and (5). Normally, when applying the sliding mode controller, a major issue is chattering phenomenon due to the use of sign function. However, in the proposed optimal control, the errors of the system are directly related to the quadratic terms of $R_{\xi}(t), L_{\xi}(t)$ (in Remark 3). Hence, when choosing parameters for $R_{\xi}(t), L_{\xi}(t)$, the chattering value is also directly assigned. The chattering phenomenon is then solved by selecting the suitable gain matrix function, as shown in (8), (13) and (14). To obtain high performance, the value of the gain matrix function should be always positive, as shown in (24). On the other hand, the use of $R_{\xi}(t), L_{\xi}(t)$ in the proposed controller is also considered as a new method, which applies initial energy to overcome the unstable states so that the stability of the system can be converged quickly and effectively. 


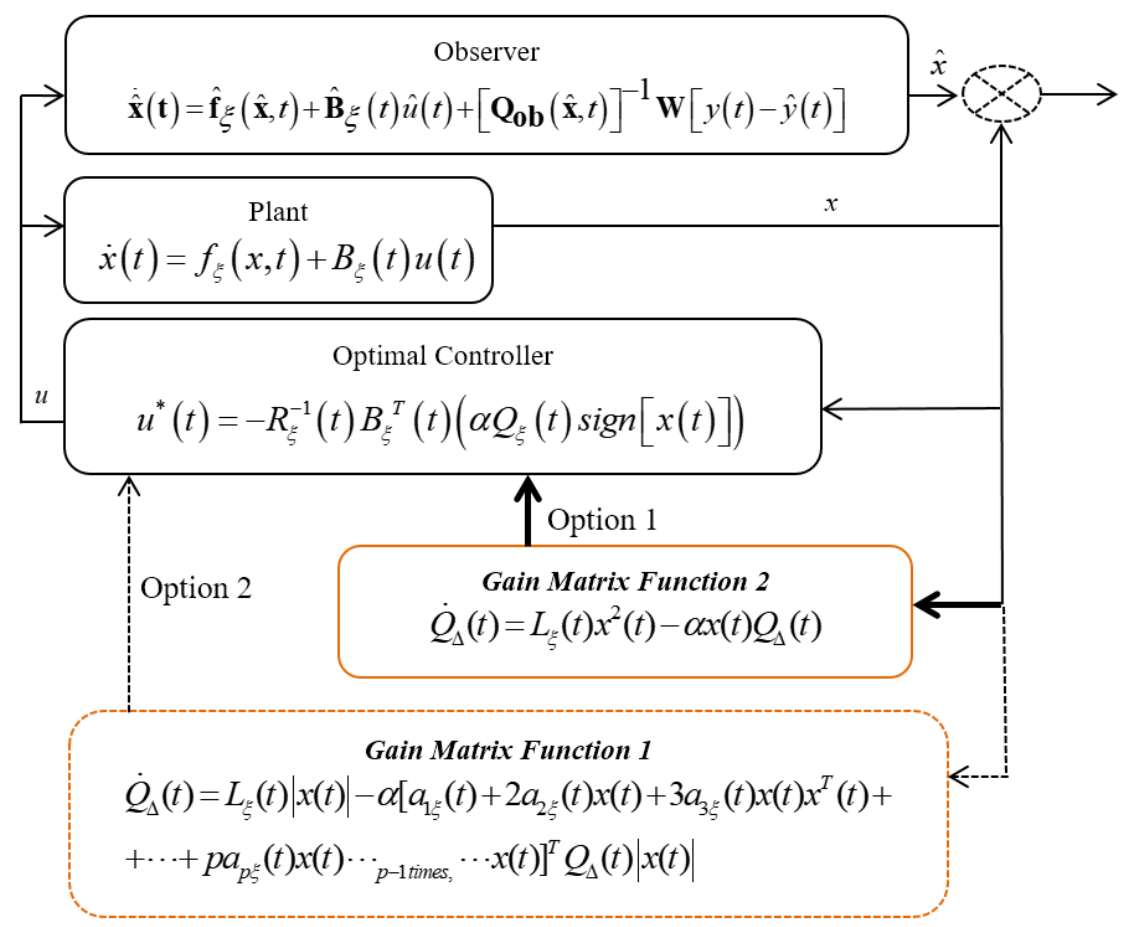

Figure 1. Block diagram of the proposed optimal control.

A schematic architecture of the proposed controller is shown in Figure 1. The output of the plant will be used for the coming calculation of the gain matrix function and compared with the observer. The observer model can be used for the progress as shown in [19]. In Figure 1, there are two options of the gain function: the gain matrix function 1 relating to the equation (14), and the gain matrix function 2 relating to the equation (8). The determination of these are carried out based on the simulation results shown in the subsequent section (Section 3).

Remark 8. In the proposed cost function (3), the matrices $R_{\xi}(t), \psi_{\xi}$, and $L_{\xi}(t)$ are chosen by user. The selection of these values depends on the dynamic property of the system. It often provides good results if the values of $R_{\xi}(t)$ and $L_{\xi}(t)$ are equal for balancing the energy of control system. Nevertheless, for complex systems, the value of $R_{\xi}(t)$ should be larger than $L_{\xi}(t)$ to increase the energy of control input. The selection of value $\psi_{\xi}$ is associated with the final state of the system. The larger the value of $\psi_{\xi}$ is, the smaller of steady state error obtains, and vice versa.

\section{Illustrative Examples}


As an illustrative example, a nonlinear system used in [11] is adopted as follows.

$$
\dot{x}_{e}(t)=0.1 x_{e}^{2}(t)+u(t) \quad x_{e}(0)=1
$$

The initial parameters used in this simulation are $t \in[0,5], R_{\xi}(t)=1, L_{\xi}(t)=1$, and the cost function is selected as:

$$
J_{2}=25\left|x_{e}(T)\right|+\frac{1}{2} \int_{0}^{T}\left(u^{2}(t)+x_{e}^{2}(t)\right) d t
$$

The main control input of the system (25) is determined by

$$
u^{*}(t)=Q_{\xi}^{*}(t) \operatorname{sign}\left[x_{e}(t)\right]
$$

The gain matrix function is solved by applying Eq. (12) as follows:

$$
\dot{Q}_{\Delta}(t)=\left|x_{e}(t)\right|-0.2 x_{e}(t) Q_{\Delta}(t)
$$

Simulation results are shown in Figure 2. In Figure 2(a), the maximum gain of the system is 5 after $4 \mathrm{~s}$, and the main control $u$ is also 5 after 4s. However, the state variables shown in Figure 2(c,d) are not stable. The system instability was occurred after applying the optimal controller [11]. These results show that the Remarks 1-3 are reasonable, and that the controller proposed in [11] cannot provide good performance.

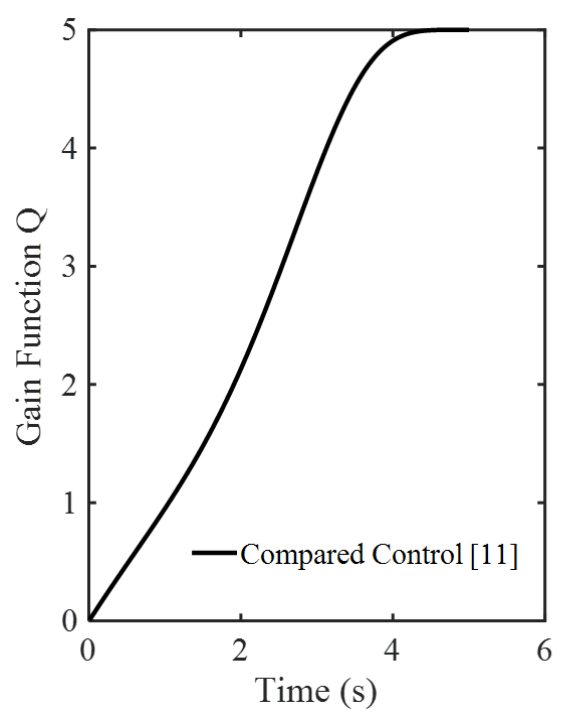

(a)

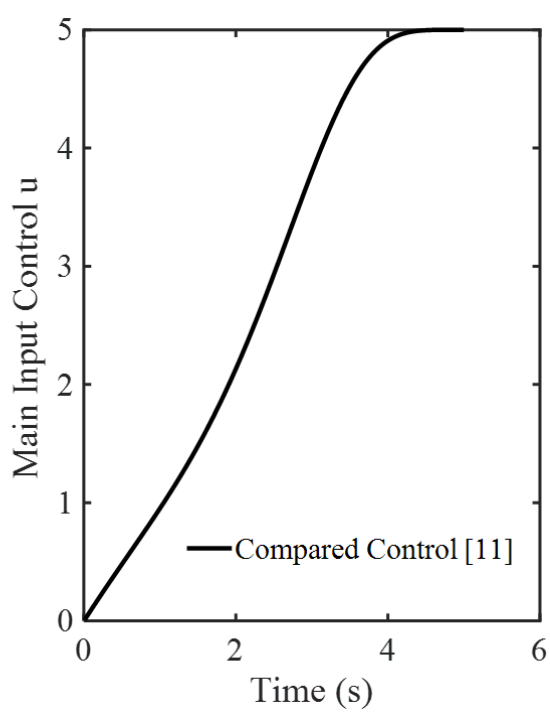

(b) 


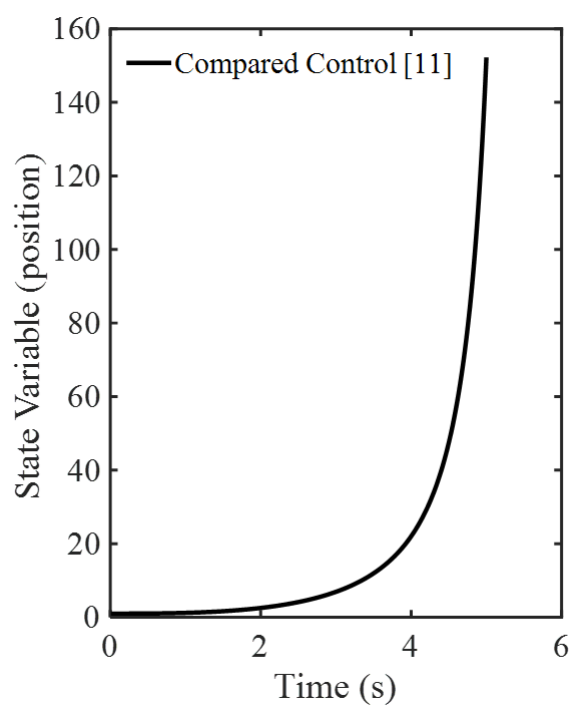

(c)

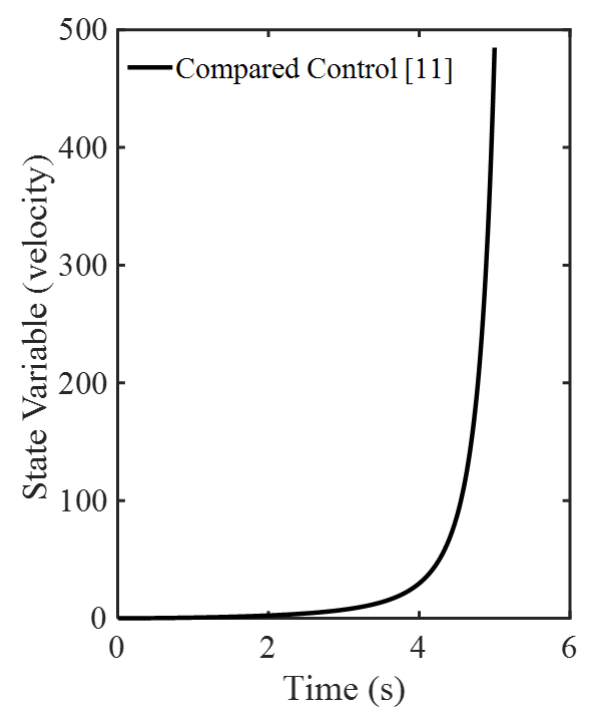

(d)

Figure 2. Simulation results of the controller in [11]: (a) gain function $Q_{\Delta}(t)$, (b) main control $u^{*}(t)$, (c) state variable (position) $x_{e}(t)$, (d) state variable (velocity) $\dot{x}_{e}(t)$.

As a next simulation, the gain matrix function shown in Eq. (14) in Remark 5 is used; the parameters of $t \in[0,300]$, and $R_{\xi}(t)=1, L_{\xi}(t)=1$ are used and the cost function is selected as follows:

$J_{2}=\frac{25\left|x_{e}(T)\right|}{\alpha}+\frac{1}{2} \int_{0}^{T}\left(u^{2}(t)+x_{e}^{2}(t)\right) d t$

The value $\alpha$ is chosen as 10 . The main control input is computed as follows:

$u^{*}(t)=-\alpha Q_{\xi}^{*}(t) \operatorname{sign}\left[x_{e}(t)\right]$

where, $Q_{\xi}{ }^{*}(t)$ is extracted from Eq. (14) as follows:

$\dot{Q}_{\Delta}(t)=\left|x_{e}(t)\right|-0.2 \alpha\left|x_{e}(t)\right| Q_{\Delta}(t)$

It is notice that the optimal controller in Eqs. (30) and (31) is called 'Proposed Control 1' to distinguish from the compared controller in [11]. In this simulation, the observer [19] is applied to emulate practical case. Simulation results are shown in Figure 3. These figures show that the proposed controller with the gain matrix function shown in Eq. (14) is better performance than the compared controller [11]. The 
magnitude of gain function is always positive, as discussed in Remark 6. A full view of the compared control [11] and the proposed control 1 is shown in Figure 4. The proposed controller obtains better performance than the compared control of [11] in all indexes.

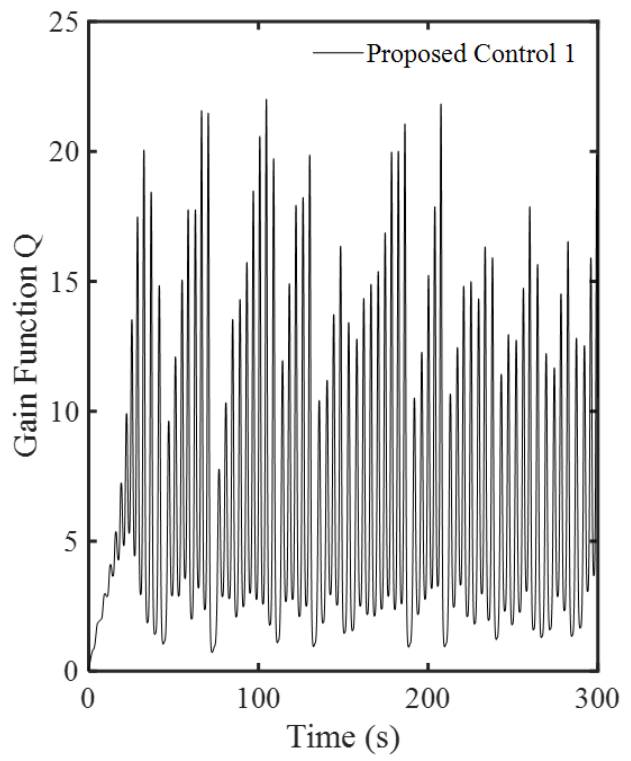

(a)

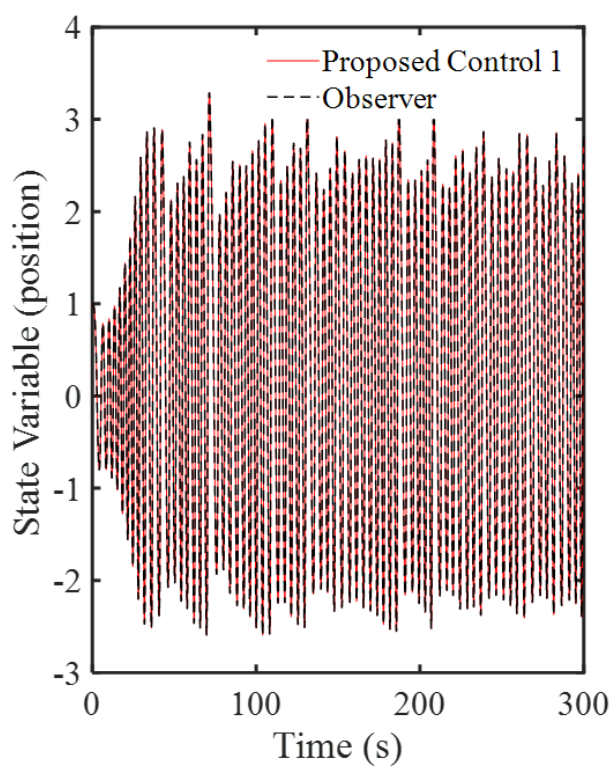

(c)

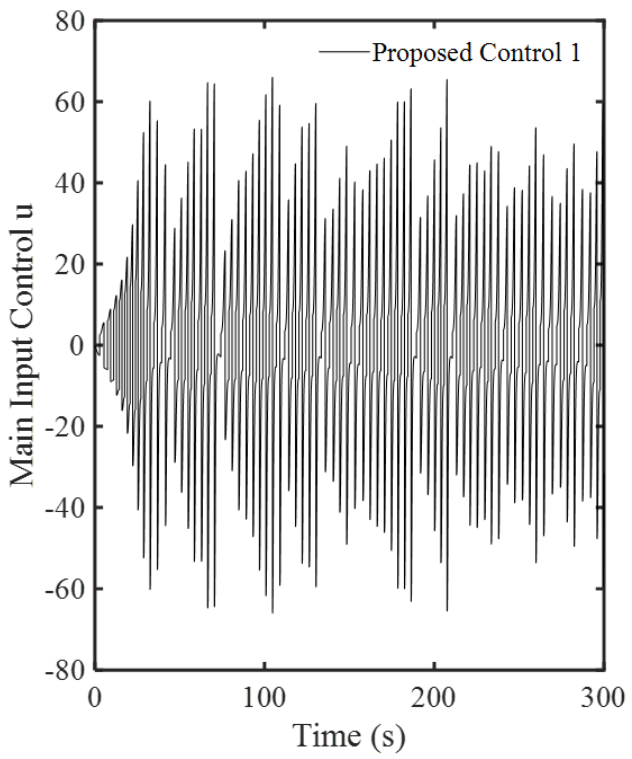

(b)

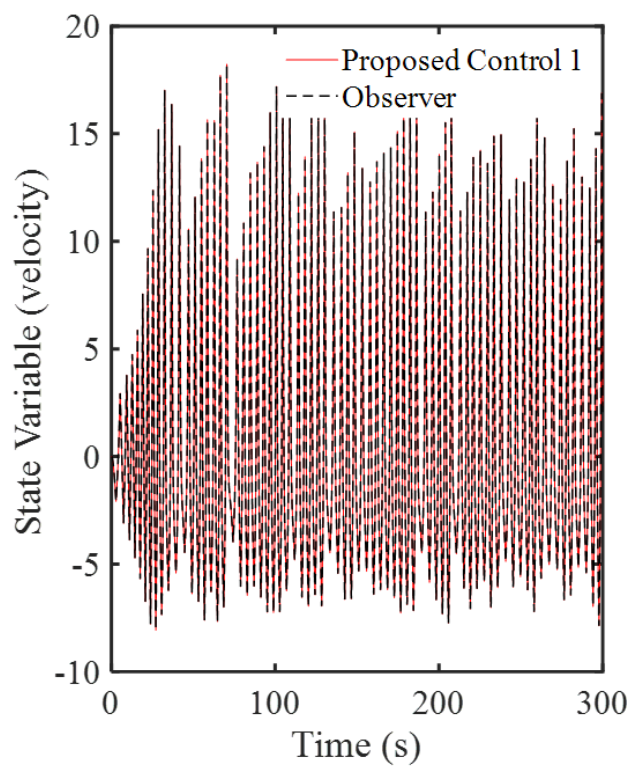

(d) 


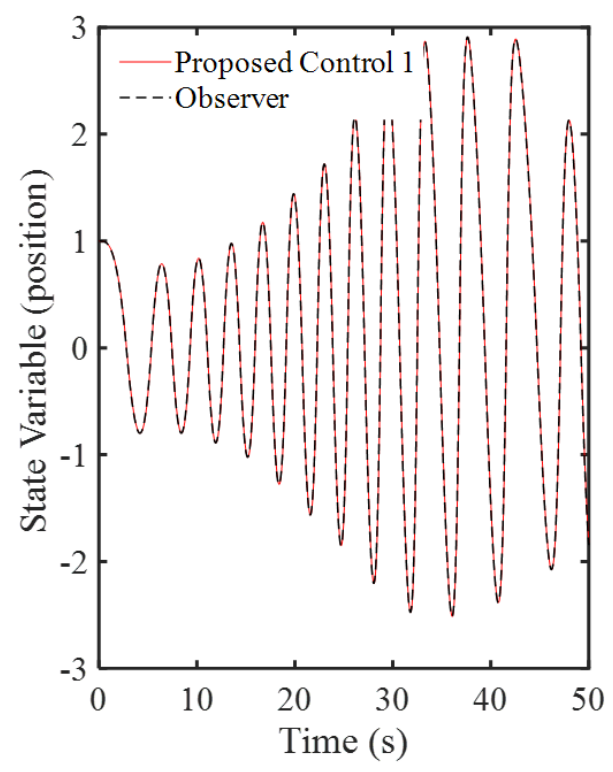

(e)

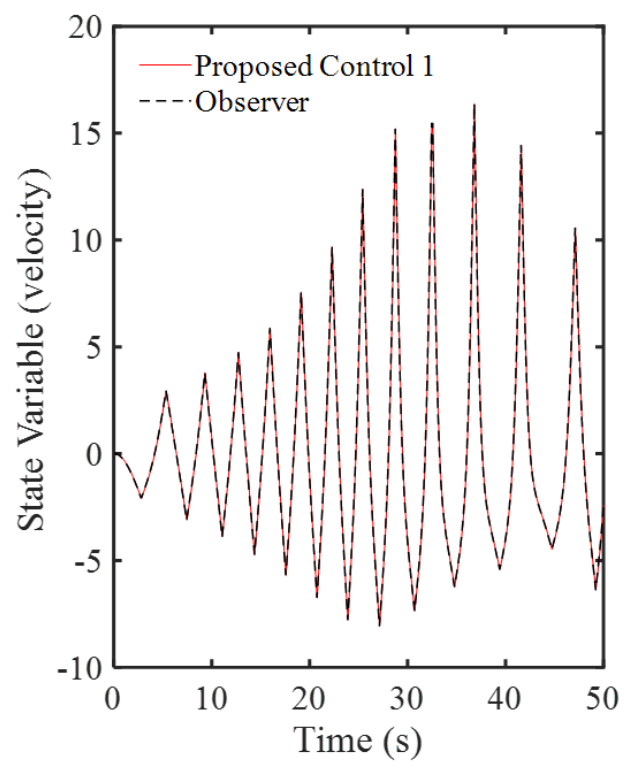

(f)

Figure 3. Simulation results of proposed control 1 following Eq. (14): (a) gain function $Q_{\Delta}(t)$, (b) main control $u^{*}(t)$, (c) state variable (position) $x_{e}(t)$, (d) state variable (velocity) $\dot{x}_{e}(t)$, (e) large view of state variable (position) $x_{e}(t)$, (f) large view of state variable (velocity) $\dot{x}_{e}(t)$.

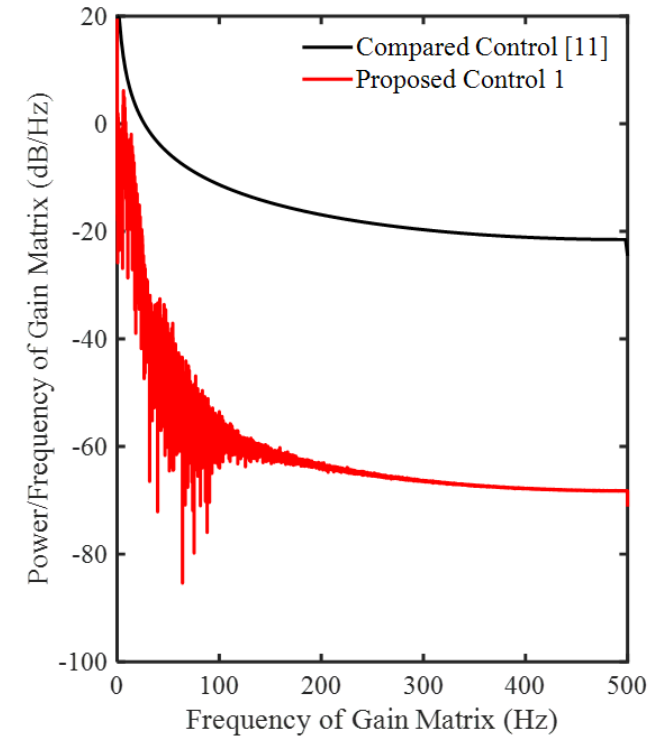

(a)

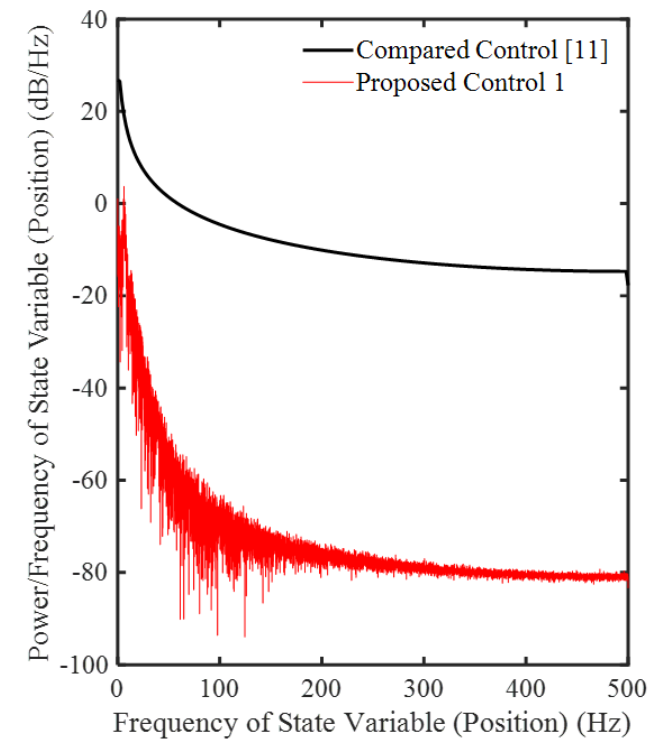

(b) 
Figure 4. Power spectral density (PSD) of proposed control 1 following Eq. (14) and compared control 2 [11]: (a) gain function $Q_{\Delta}(t)$, (b) state variable (position) $x_{e}(t)$.

Now, in order to investigate the effect of the gain matrix function, the following equation, which is a form of Eq. (13), is chosen for the simulation

$\dot{Q}_{\Delta}(t)=L_{\xi}(t) x_{e}^{2}(t)-\alpha x_{e}(t) Q_{\Delta}(t)$

The parameters of $t \in[0,300]$ and $R_{\xi}(t)=1, L_{\xi}(t)=1$ are used. This controller is called 'Proposed Control 2'. In this simulation, the observer [19] is also applied. The simulation results are shown in Figure 5. After applying the proposed method 2, the magnitude of all parameters is dramatically decreased. This result proves that the Proposed Control 2 provides better control performance than the Proposed Control 1. The energy used for the control 2 is shown in Figure 5(b). Thus, using the Proposed Control 2, some benefits including energy saving and high tracking performance are achieved. It should be mentioned that besides the aforementioned benefits of the proposed optimal controller, the variation of $R_{\xi}(t) L_{\xi}(t)$ can be easily adapted to provide high control performance with less energy consumption.

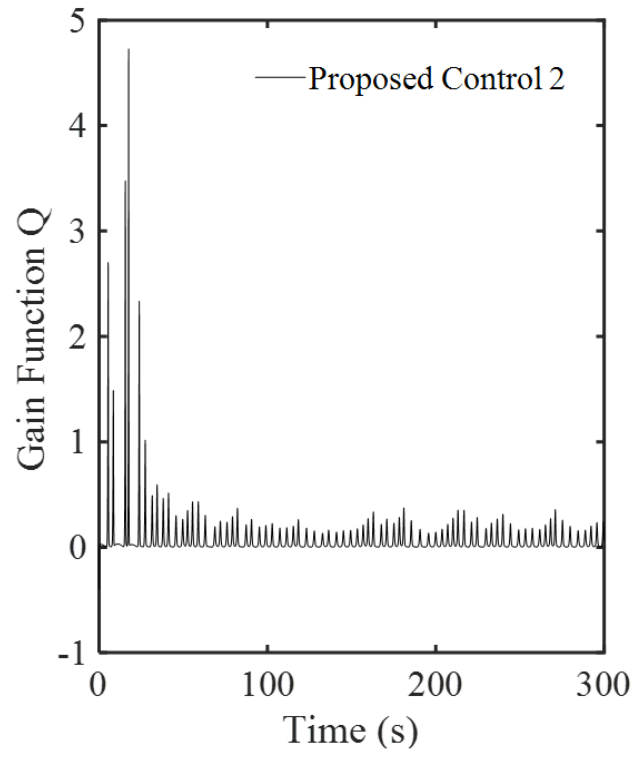

(a)

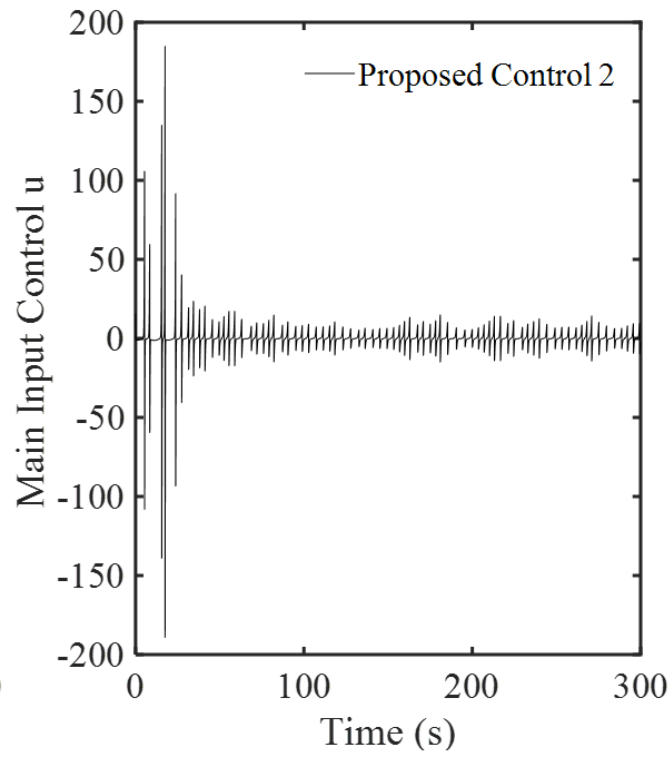

(b) 


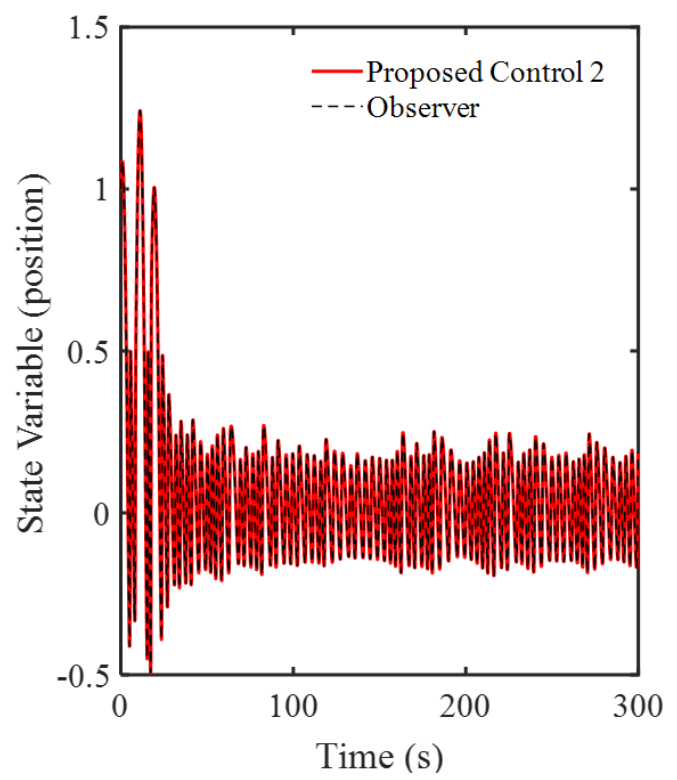

(c)

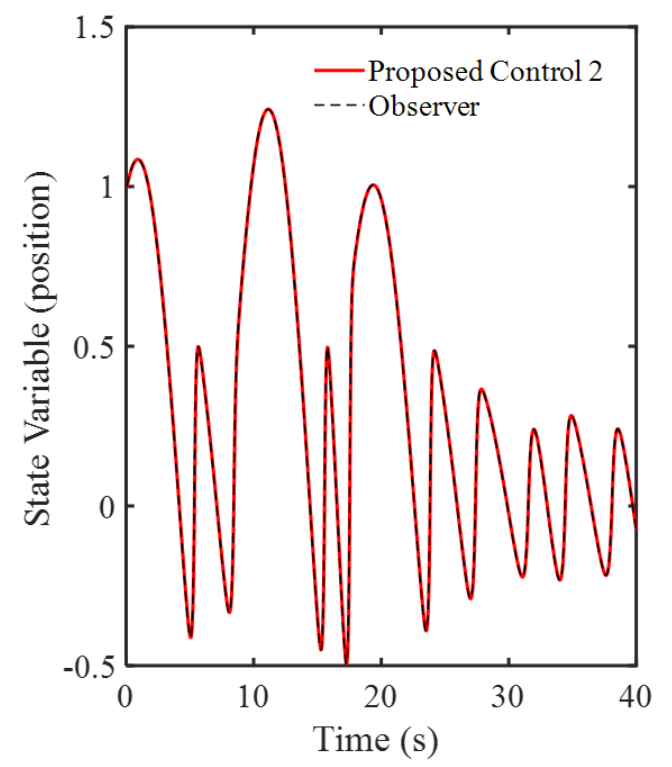

(e)

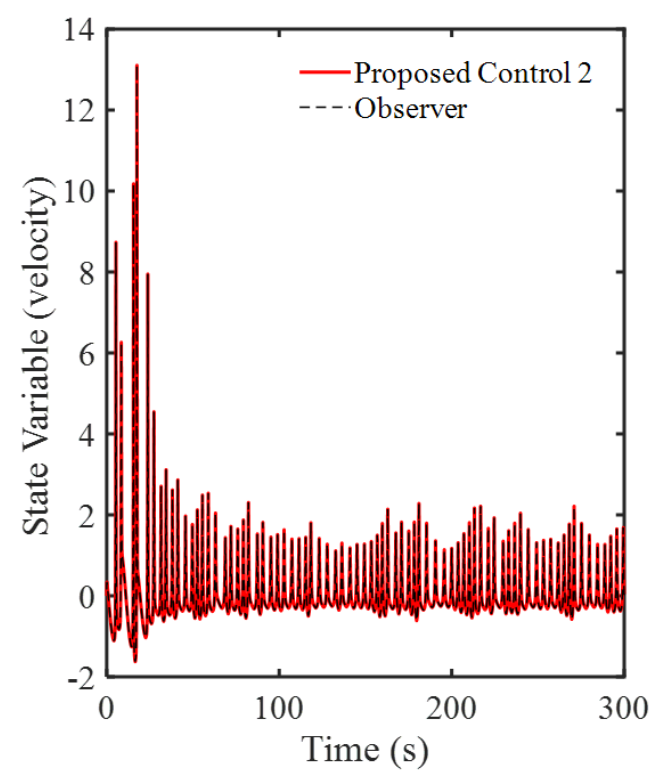

(d)

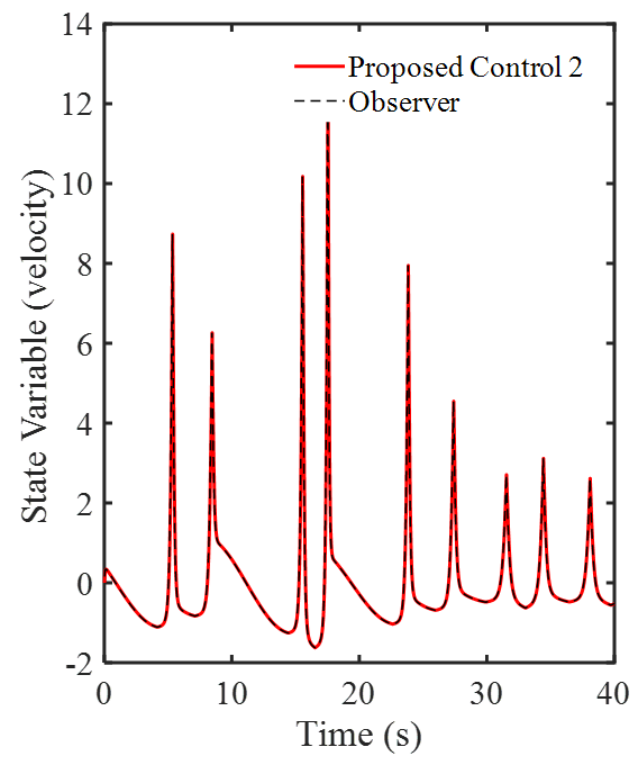

(f)

Figure 5. Simulation results of proposed control 2 following Eq. (8): (a) gain function $Q_{\Delta}(t)$, (b) main control $u^{*}(t)$, (c) state variable (position) $x_{e}(t)$, (d) state variable (velocity) $\dot{x}_{e}(t)$, (e) large view of state variable (position) $x_{e}(t)$, (f) large view of state variable (velocity) $\dot{x}_{e}(t)$.

Now, in order to investigate the effects of the variations of $R_{\xi}(t) L_{\xi}(t)$ on the system performances, the values of $R_{\xi}(t), L_{\xi}(t)$ are assumed to be varied from $R_{\xi}(t)=1, L_{\xi}(t)=1$ to $R_{\xi}(t)=1.5, L_{\xi}(t)=1.2$. 
The results of this simulation are shown in Figures 6. In Figure 6, the gain function $Q_{\Delta}(t)$ and the main control input $u^{*}(t)$ are less than the values shown in Figure 5. The noise shown in Figure 6 is also less than the value shown in Figure 5. This simulation result shows that the noise is decreased when the value of $R_{\xi}(t), L_{\xi}(t)$ is changed. In addition, the energy of control input is also less than the case of $R_{\xi}(t)=1, L_{\xi}(t)=1$. This is one of the most eminent advantages of the Proposed Control 2. This phenomenon is clearly shown in Figure 7 based on power spectral density (PSD) criteria. Hence, the application of the Proposed Control 2 is better than the Proposed Control 1 in terms of the stability of the system.

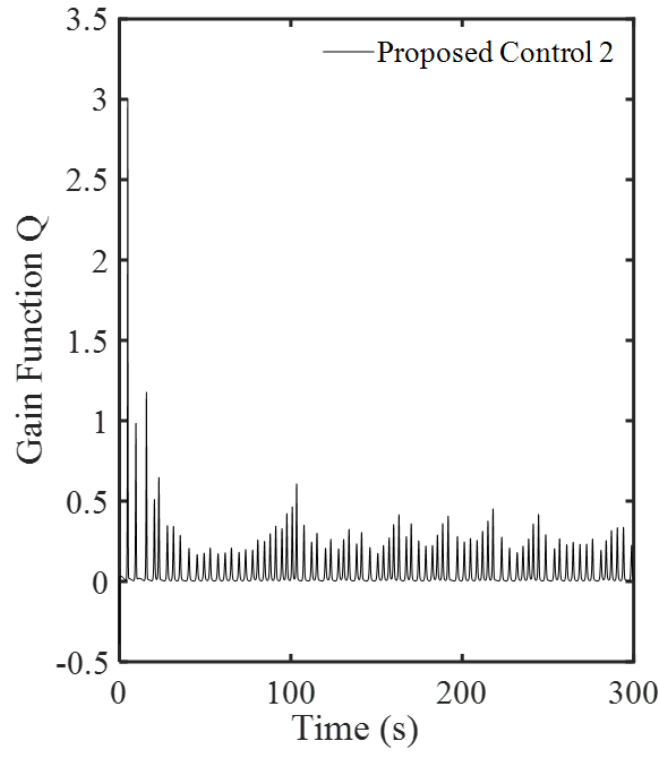

(a)

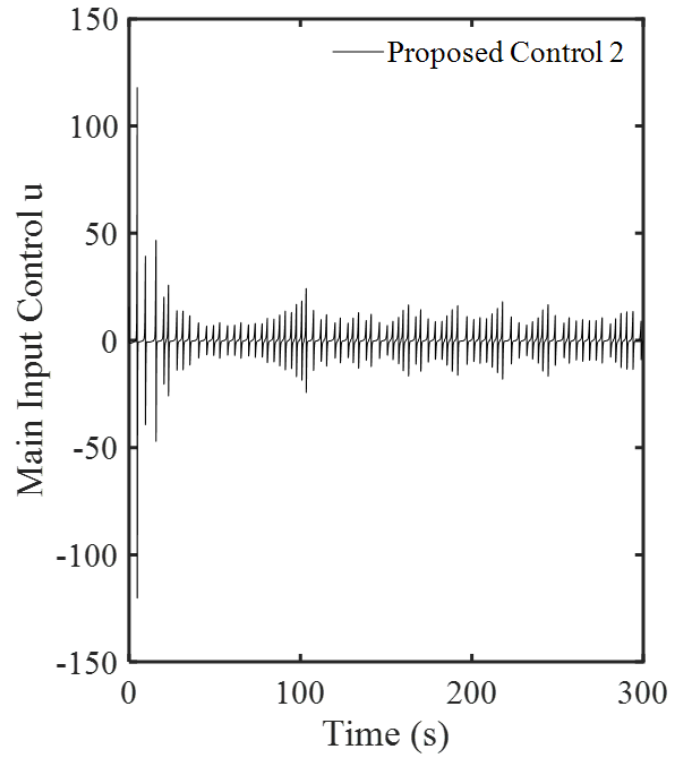

(b) 


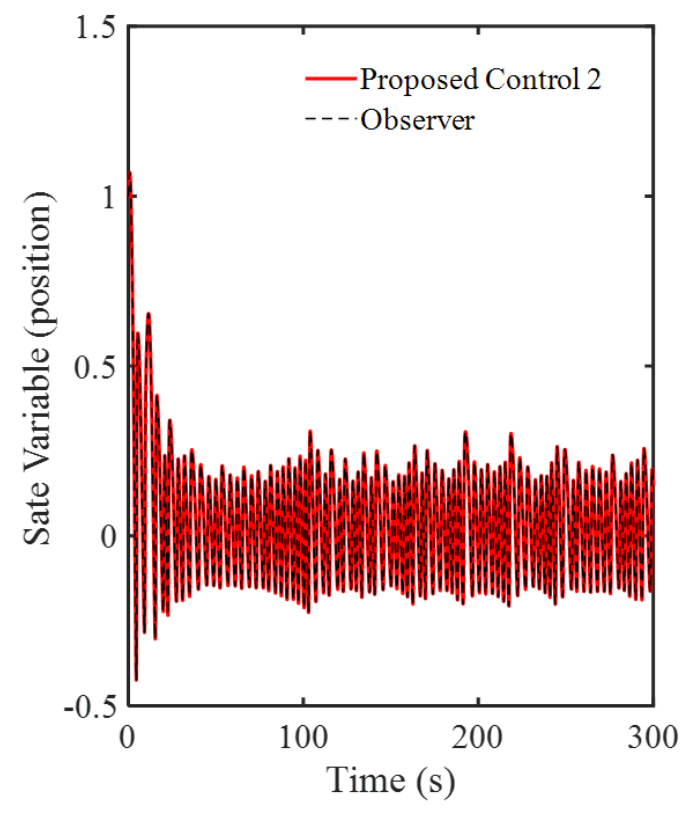

(c)

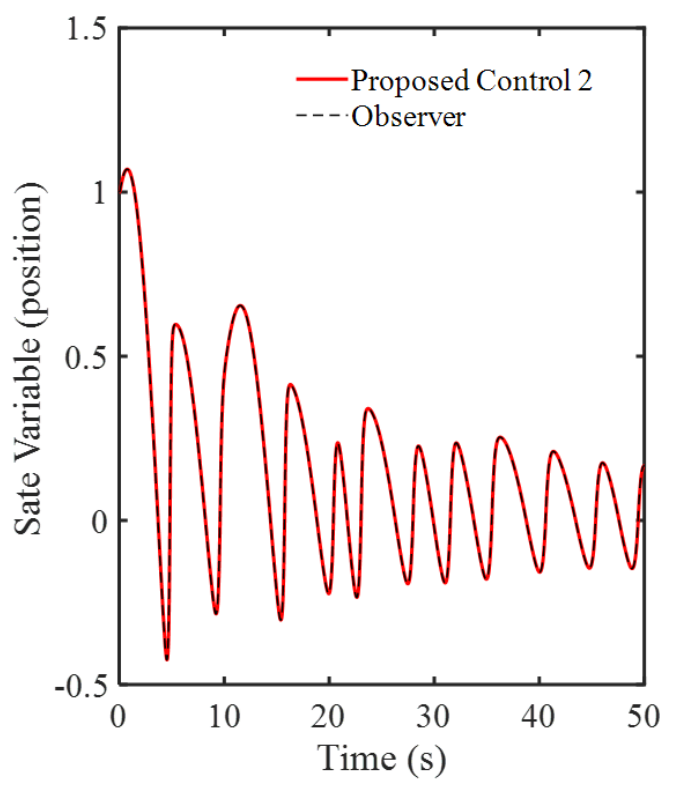

(e)

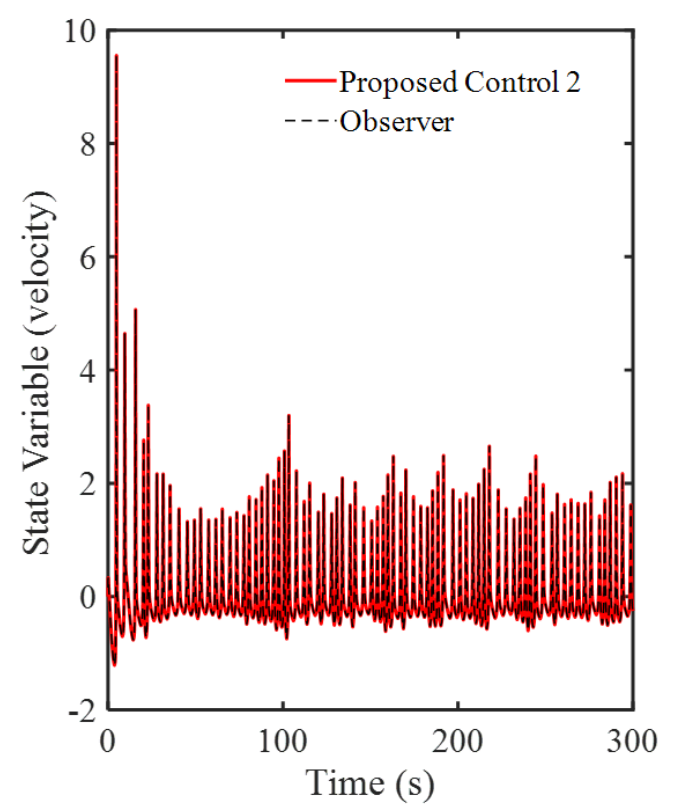

(d)

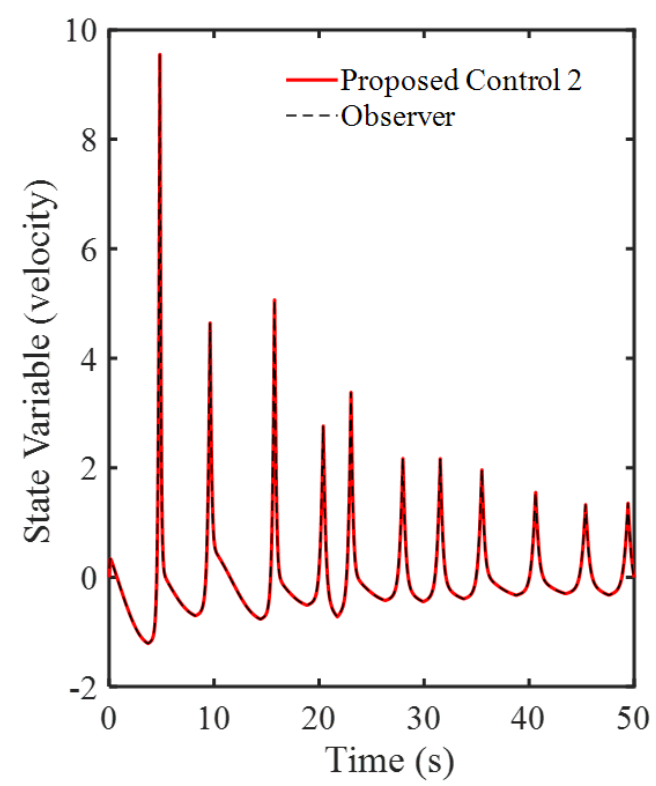

(f)

Figure 6. Simulation results of proposed control 2 following Eq. (8) when $R_{\xi}(t)=1.5, L_{\xi}(t)=1.2$ : (a) gain function $Q_{\Delta}(t)$, (b) main control $u^{*}(t)$, (c) state variable (position) $x_{e}(t)$, (d) state variable (velocity) $\dot{x}_{e}(t),(\mathrm{e})$ large view of state variable (position) $x_{e}(t)$, (f) large view of state variable (velocity) $\dot{x}_{e}(t)$. 


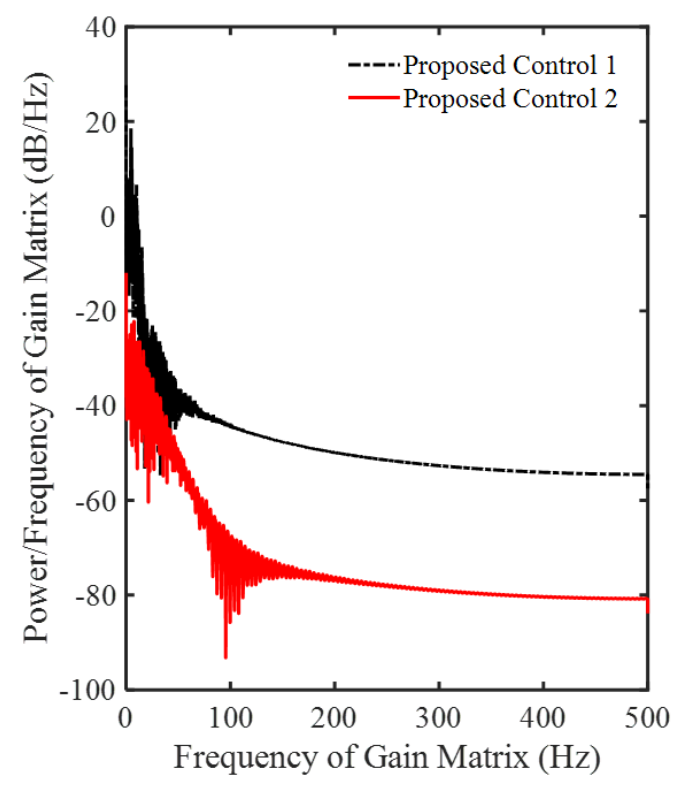

(a)

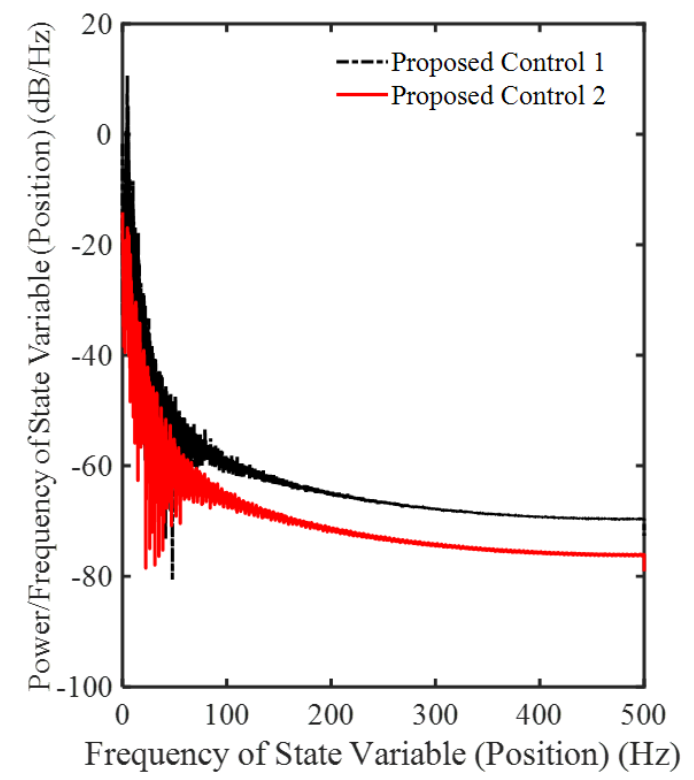

(b)

Figure 7. Power spectral density (PSD) of proposed control 1 following Eq. (14) and proposed control 2 following Eq. (8): (a) gain function $Q_{\Delta}(t)$, (b) state variable (position) $x_{e}(t)$.

\section{Application to Vibration Control}

In order to demonstrate the effectiveness of the proposed controller, a practical control system considered in [16], [17], and [18] is adopted and vibration control performance is evaluated by implementing the Proposed Control 2. The control system considered in the previous works is the vehicle seat suspension with controllable magnetorheological (MR) damper. The mechanical model is shown in Figure 8. The governing equations of the system are derived as follows:

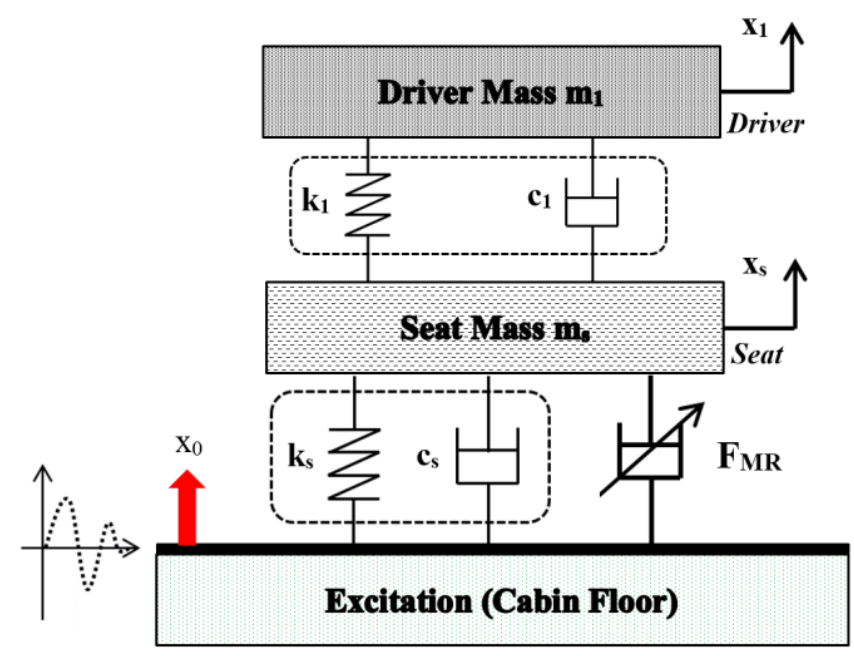

Figure 8. Mechanical model of the seat suspension 


$$
\begin{aligned}
& m_{s} \ddot{x}_{s}=-k_{s}\left(x_{s}-x_{0}\right)-c_{s}\left(\dot{x}_{s}-\dot{x}_{0}\right)+k_{1}\left(x_{1}-x_{s}\right)+c_{1}\left(\dot{x}_{1}-\dot{x}_{s}\right)+F_{M R} \\
& m_{1} \ddot{x}_{1}=-k_{1}\left(x_{1}-x_{s}\right)+c_{1}\left(\dot{x}_{1}-\dot{x}_{s}\right)
\end{aligned}
$$

The above equations are then rewritten using the state variables as follows.

$$
\begin{gathered}
\dot{x}_{11}=\dot{x}_{s}=x_{22} \\
\dot{x}_{22}=f_{11}\left(x_{11}, x_{22}, x_{33}, x_{44}\right)+g_{11}\left(x_{11}, x_{22}, x_{33}, x_{44}\right) u \\
\dot{x}_{33}=\dot{x}_{1}=x_{44} \\
\dot{x}_{44}=f_{22}\left(x_{11}, x_{22}, x_{33}, x_{44}\right)
\end{gathered}
$$

where,

$$
\begin{aligned}
& f_{11}\left(x_{11}, x_{22}, x_{33}, x_{44}\right)=-\frac{k_{s}}{m_{s}}\left(x_{11}-x_{0}\right)-\frac{c_{s}}{m_{s}}\left(x_{22}-\dot{x}_{0}\right)+\frac{k_{1}}{m_{s}}\left(x_{33}-x_{11}\right)+\frac{c_{1}}{m_{s}}\left(x_{44}-x_{22}\right), \\
& g_{11}\left(x_{11}, x_{22}, x_{33}, x_{44}\right)=\frac{1}{m_{s}}, u=F_{M R}, f_{22}\left(x_{11}, x_{22}, x_{33}, x_{44}\right)=-\frac{k_{1}}{m_{1}}\left(x_{33}-x_{11}\right)-\frac{c_{1}}{m_{1}}\left(x_{44}-x_{22}\right) .
\end{aligned}
$$

Here, $x_{11}, x_{22}, x_{33}, x_{44}$ are variables with respect to $x_{s}$ and $x_{1}$ of the system. The calculated result of $u=F_{M R}$ must be converted to the input signal to generate the required magnetic field for MR damper.

This work is done by applying the relationship given as follows:

$$
F_{M R}=\left(c_{a}+c_{b} \vartheta\right)\left(x_{44}-x_{22}\right)+k_{0}\left(x_{33}-x_{11}\right)+\left(\alpha_{a}+\alpha_{b} \vartheta\right) \phi
$$

where, $\dot{\phi}=-\kappa\left|x_{44}-x_{22}\right|+\lambda_{d}\left(x_{44}-x_{22}\right)|\phi|+\varphi_{d}\left(x_{44}-x_{22}\right)$. Using Eq. (36), the voltage of $\vartheta$ to be applied to MR damper is calculated as follows:

$$
\vartheta=\frac{F_{M R}-\left[c_{a}\left(x_{44}-x_{22}\right)+k_{0}\left(x_{33}-x_{11}\right)+\alpha_{a} \phi\right]}{c_{b}\left(x_{44}-x_{22}\right)+\alpha_{b} \phi}
$$

It is notice that the voltage $\vartheta$ will be converted to the input current related to the damping force of MR damper. The parameters of the seat suspension system are listed in Tables 1-3. The parameters of $t \in[0,300], R_{\xi}(t)=20, L_{\xi}(t)=20, Q_{\Delta}(0)=0.1$ and $\gamma=100$ are chosen.

Table 1. Parameters of MR seat suspension system [26]

\begin{tabular}{l|l}
\hline Parameter & Value \\
\hline Mass of seat $m_{s}$ & $27 \mathrm{~kg}$ \\
\hline Mass of driver $m_{1}$ & $77 \mathrm{~kg}$ \\
Stiffness coefficient of seat $k_{s}$ & $17830 \mathrm{~N} / \mathrm{m}$ \\
\hline Stiffness coefficient of torso $k_{1}$ & $49340 \mathrm{~N} / \mathrm{m}$ \\
\hline
\end{tabular}




\begin{tabular}{ll} 
Damping coefficient of seat $c_{s}$ & $1500 \mathrm{Ns} / \mathrm{m}$ \\
\hline Damping coefficient of torso $c_{1}$ & $2475 \mathrm{Ns} / \mathrm{m}$ \\
\hline
\end{tabular}

Table 2. Parameters of MR damper

\begin{tabular}{l|l}
\hline Parameter & Value \\
\hline Stiffness of gas chamber $k_{e}$ & $94.6166 \mathrm{~N} / \mathrm{m}$ \\
\hline Damping coefficient $c_{e}$ & $794.4681 \mathrm{Ns} / \mathrm{m}$ \\
\hline Piston area $A_{p}$ & $0.00113354 \mathrm{~m}^{2}$ \\
\hline Piston rod area $A_{r}$ & $0.0000785 \mathrm{~m}^{2}$ \\
\hline Gap of magnetic pole $h$ & $0.01 \mathrm{~m}$ \\
\hline Length of magnetic pole $L$ & $0.05 \mathrm{~m}$ \\
\hline MR inherent coefficient $\alpha_{1}$ & 26.77185 \\
\hline MR inherent coefficient $\alpha_{2}$ & 0.60444 \\
\hline
\end{tabular}

Table 3. Parameters to calculate the applied current of MR damper

\begin{tabular}{l|l}
\hline Parameter & Value \\
\hline Linear spring stiffness $k_{0}$ & $0 \mathrm{~N} / \mathrm{m}$ \\
\hline Viscous damping coefficient $c_{a}$ & $990 \mathrm{Ns} / \mathrm{m}$ \\
\hline Viscous damping coefficient influenced by $v c_{b}$ & $3095 \mathrm{Ns} / \mathrm{m} \mathrm{V}$ \\
\hline Stiffness of $\varphi_{d} \alpha_{a}$ & $545 \mathrm{~N} / \mathrm{m}$ \\
\hline Stiffness of $\varphi_{d}$ influenced by $v \alpha_{b}$ & $620 \mathrm{~N} / \mathrm{mV}$ \\
\hline Positive parameter of hysteresis loop $\phi$ & 4 \\
\hline Positive parameter of hysteresis loop $\kappa$ & 48 \\
\hline Positive parameter of hysteresis loop $\lambda$ & 48 \\
\hline
\end{tabular}




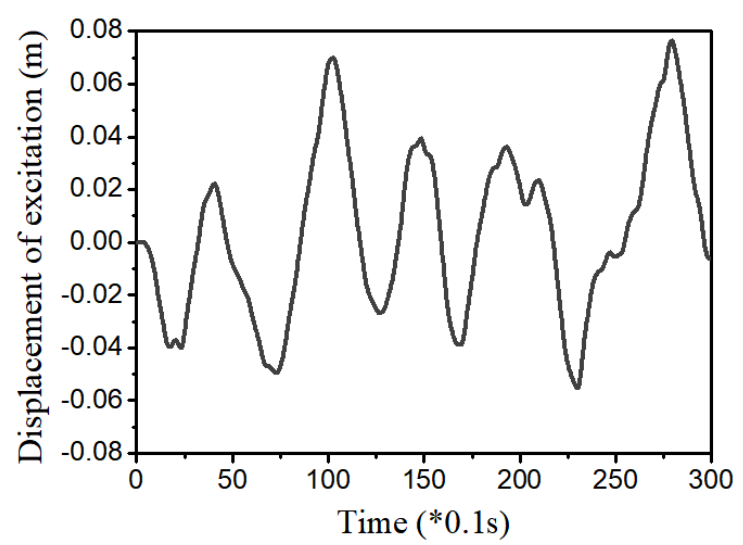

Figure 9. Random bump road excitation.

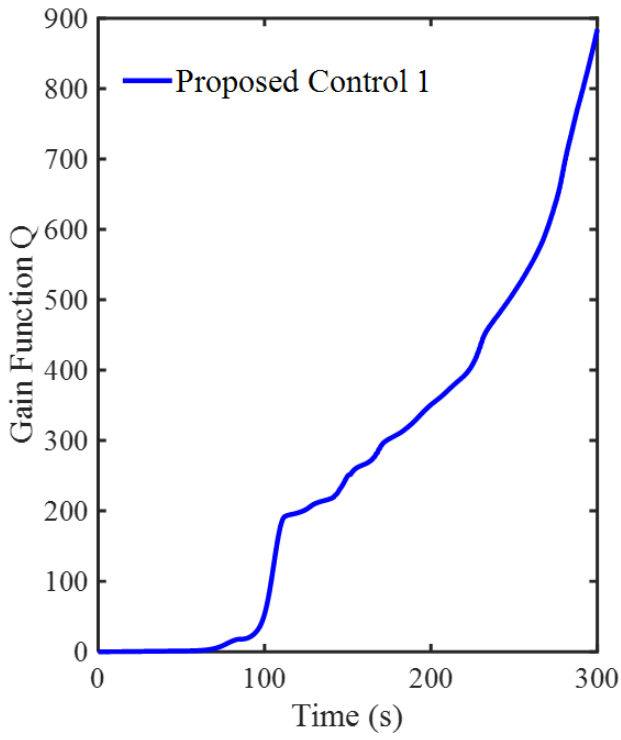

(a)

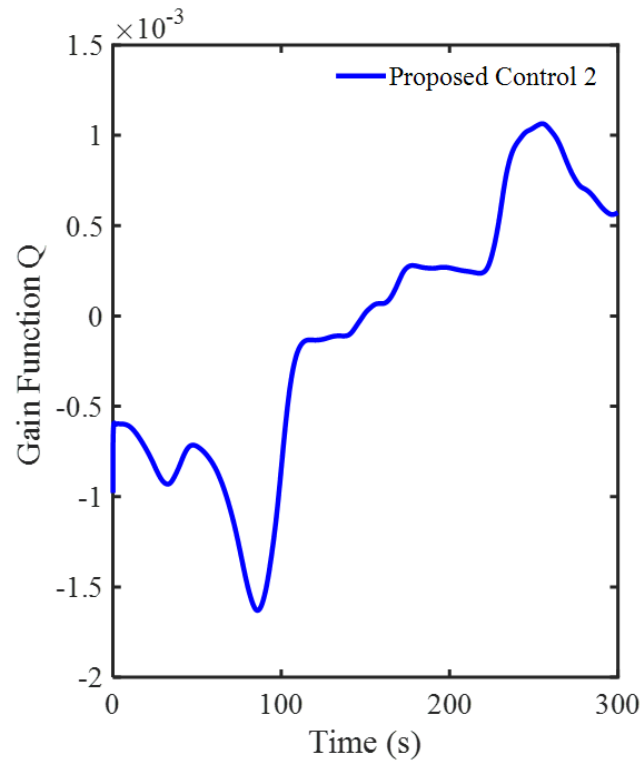

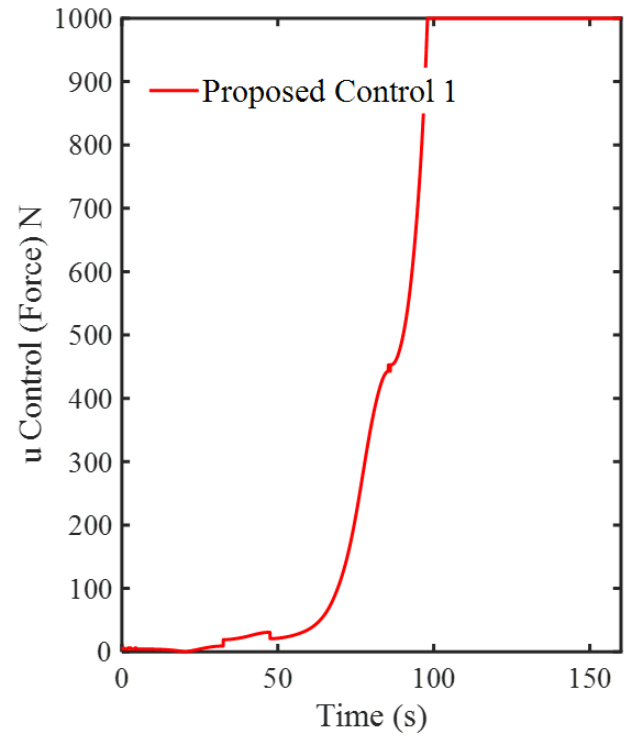

(b)

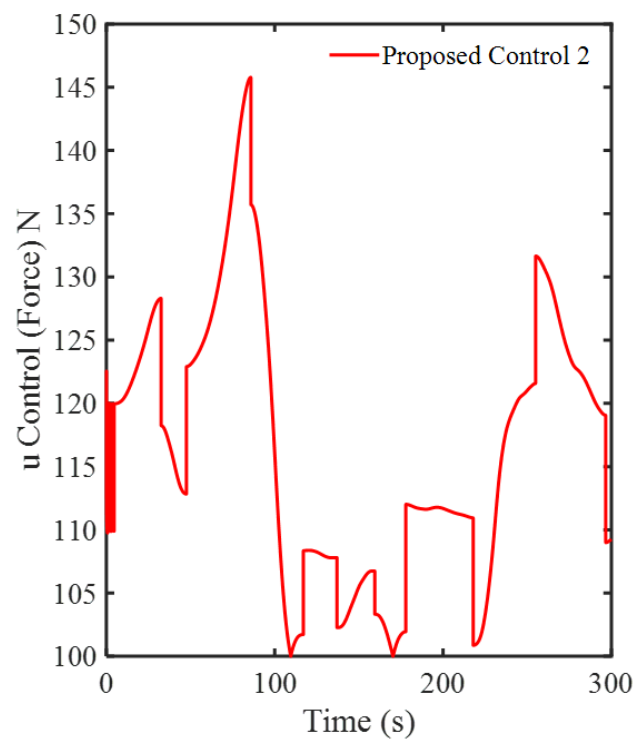


Figure 10. Simulation results of gain function $Q_{\Delta}(t)$ and main control $u^{*}(t)$ of random bump road excitation: (a) proposed control 1, (b) proposed control 2.

Based on the parameters given in Tables 1-3, the simulation is undertaken using both the Proposed Control 1 and the Proposed Control 2. As for the excitation to the seat damper, a random bump road excitation shown in Figure 9 is used and simulation results are shown in Figures 10-12. In Figures 10(a, c), the gain value $Q_{\Delta}(t)$ is always changed and its final value is the positive number, as predicted in Remark 6 . The main control input $u^{*}(t)$ shown in Figures $10(\mathrm{~b}, \mathrm{~d})$ is also changed corresponding to the variation of vibration. The results of system states are shown in Figures 11. The results in Figure 10 shown that the vibration of the seat system is converged to the boundary of $(-0.005,0.005)$. The vibration of seat is decreased with large difference after using both the Proposed Control 1 and the Proposed Control 2. However, the response of the Proposed Control 1 is not good as shown in Figure 11(d). These results prove that the Proposed Control 2 is very effective for vibration control of the vehicle seat suspension subjected to external disturbances. This result can be seen in Figure 12 based on PSD indexes. It I also notice that the noise of the Proposed Control 1 is larger than the Proposed Control 2.

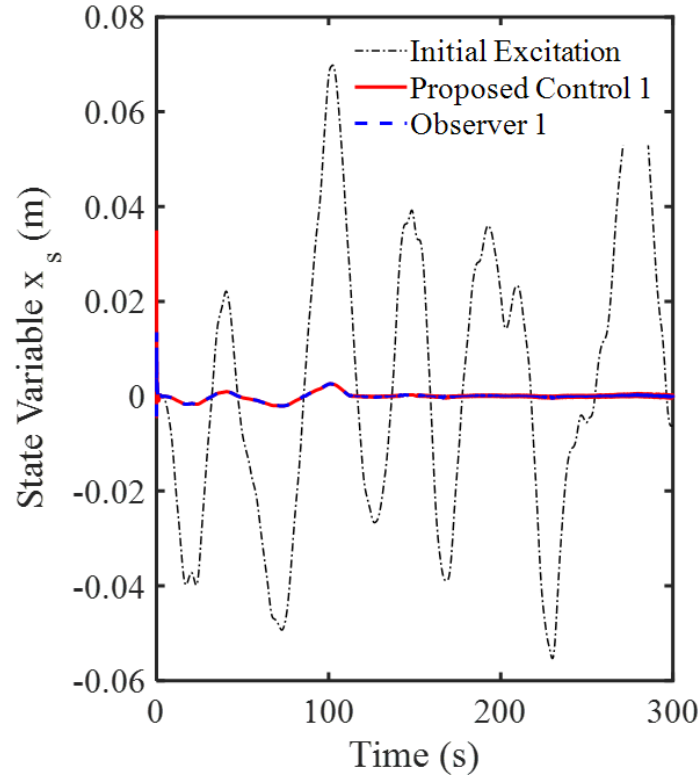

(a)

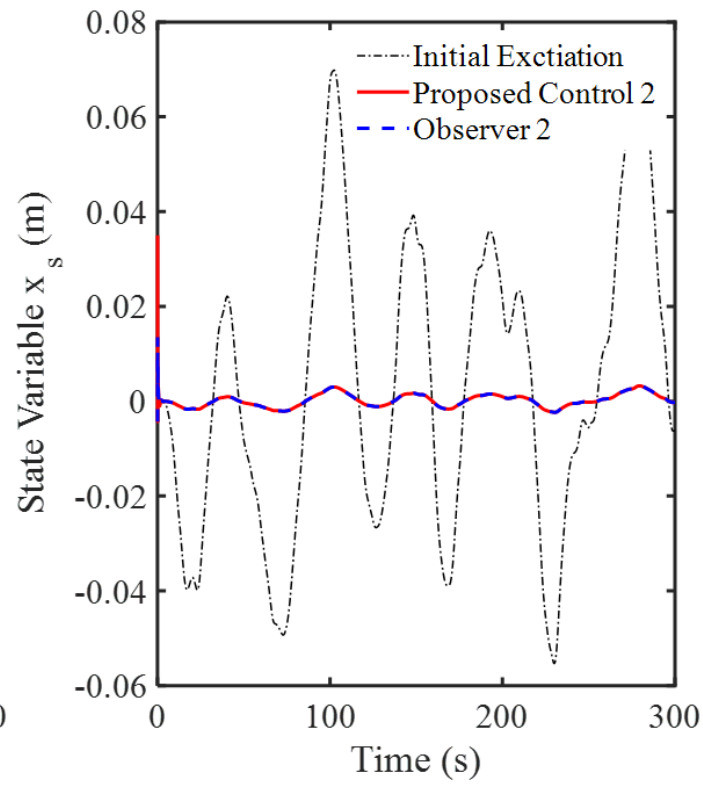

(b) 


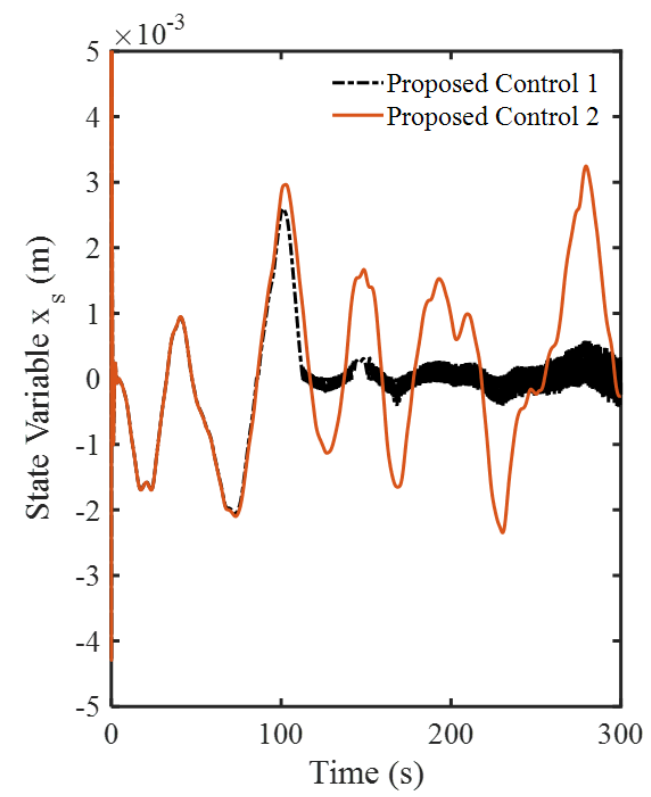

(c)

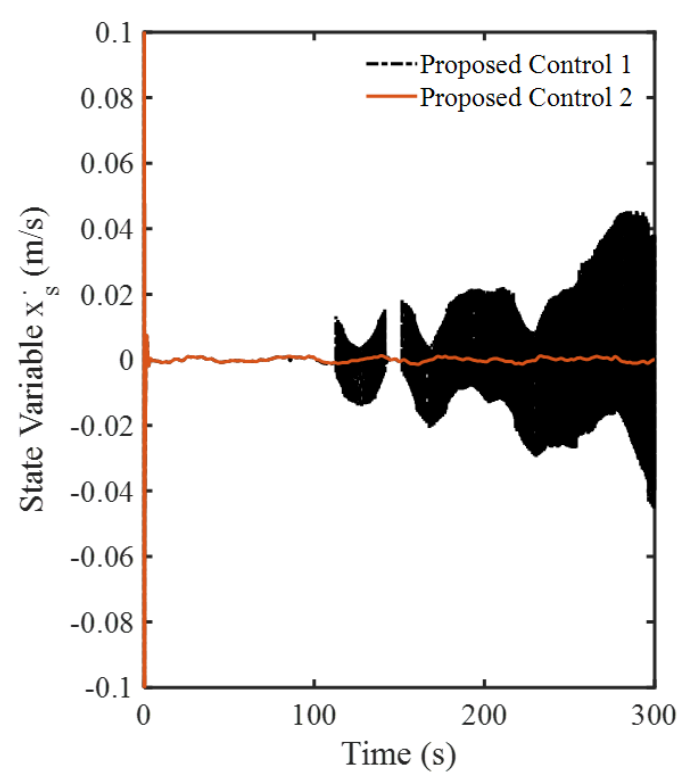

(d)

Figure 11. Simulation results of proposed control 2 following Eq. (8) of random bump road excitation of seat system: (a) state variable of proposed control $1 x_{s}(t)$, (b) state variable of proposed control $2 x_{s}(t)$ , (c) state variable $x_{s}(t)$ of proposed control 1 and 2, (d) state variable $\dot{x}_{s}(t)$ of proposed control 1 and 2.

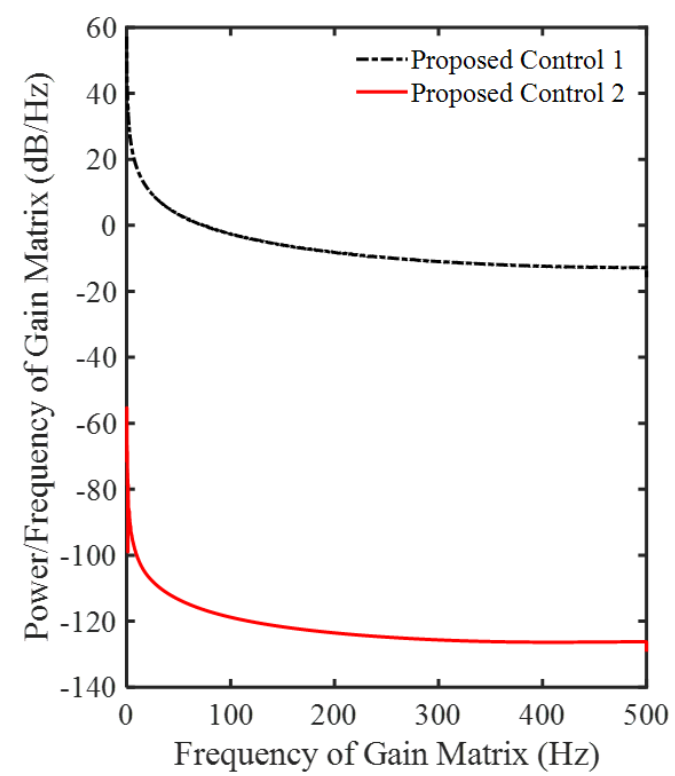

(a)

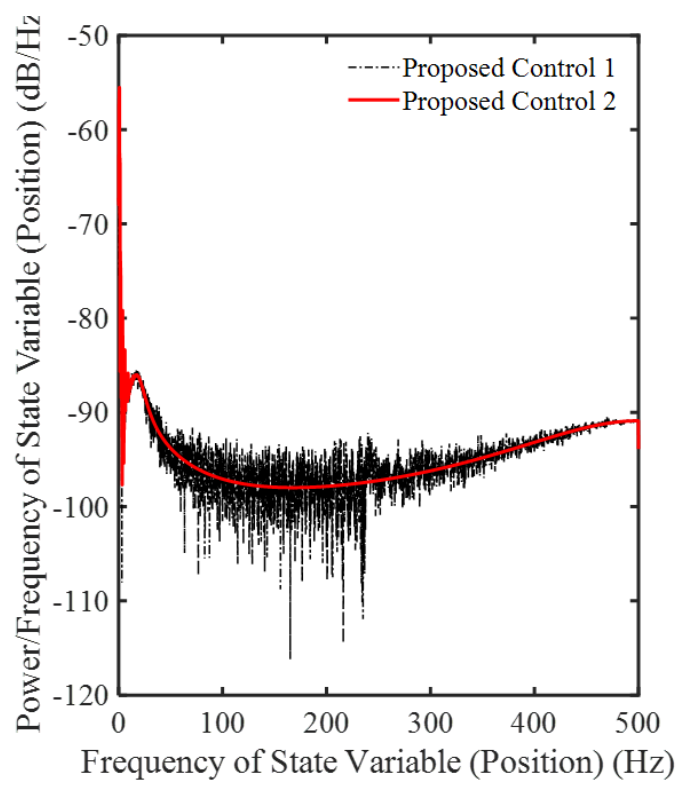

(b)

Figure 12. Power spectral density (PSD) of proposed control 1 following Eq. (14) and proposed control 2 following Eq. (8): (a) gain function $Q_{\Delta}(t)$, (b) state variable (position) $x_{e}(t)$. 
Based on the above analyses, the proposed control 2 is then compared with the previous studies [23], [24], and [25]. In the other words, the comparison is made between the controllers in [23, 24] and the controller in [25]. The main parameters of the controllers [23], [24] and [25] are listed in Table 4, Table 5, respectively. The results are shown in Figure 13 with PSD distributions. This figure shows that the proposed controller is better than other controllers, and the power of the proposed controller is also less than the compared controllers in [23], [24], and [25]. This outstanding point also proves that the gain matrix approach can save the energy and guarantee the stability of the system. This method is a valuable supplement for controlling the complex system subjected to severe disturbance.

Table 4. Parameters of simulation $[23,24]$

\begin{tabular}{l|l|l}
\hline \multicolumn{1}{c|}{ Description } & \multicolumn{1}{|c|}{ Symbol } & \multicolumn{1}{c}{ Value } \\
\hline Input control & {$\left[\mathrm{u}_{\min }, \mathrm{u}_{\max }\right]$} & {$[0 ; 4](\mathrm{A})$} \\
\hline Initial matrix & $\mathbf{R}, \mathbf{r}$ & Unit matrix \\
\hline Matrices (the running cost) & $\mathbf{L}$ & $10 ; 10$ \\
\hline Parameter & $\phi_{x}$ & 1 \\
\hline Parameter & $\phi_{x x}$ & 10 \\
\hline
\end{tabular}

Table 5. Parameters of simulation [25]

\begin{tabular}{|c|c|c|}
\hline Description & Symbol & Value \\
\hline Lumped matrix & $\xi$ & 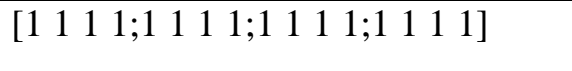 \\
\hline System matrix & $\mathbf{C}$ & {$[1 ; 0 ; 0 ; 0]$} \\
\hline Matrix (cost function) & $\mathbf{R}$ & [1] \\
\hline Matrix & $\mathbf{P}$ & $1 \mathrm{e} 5^{*}[1111 ; 1111 ; 1111 ; 1111]$ \\
\hline Matrix & $\mathbf{P}_{\mathrm{t}}$ & Solved equation (25) [25] \\
\hline Desired value & $\mathrm{y}_{\mathrm{d}}$ & 0 \\
\hline
\end{tabular}




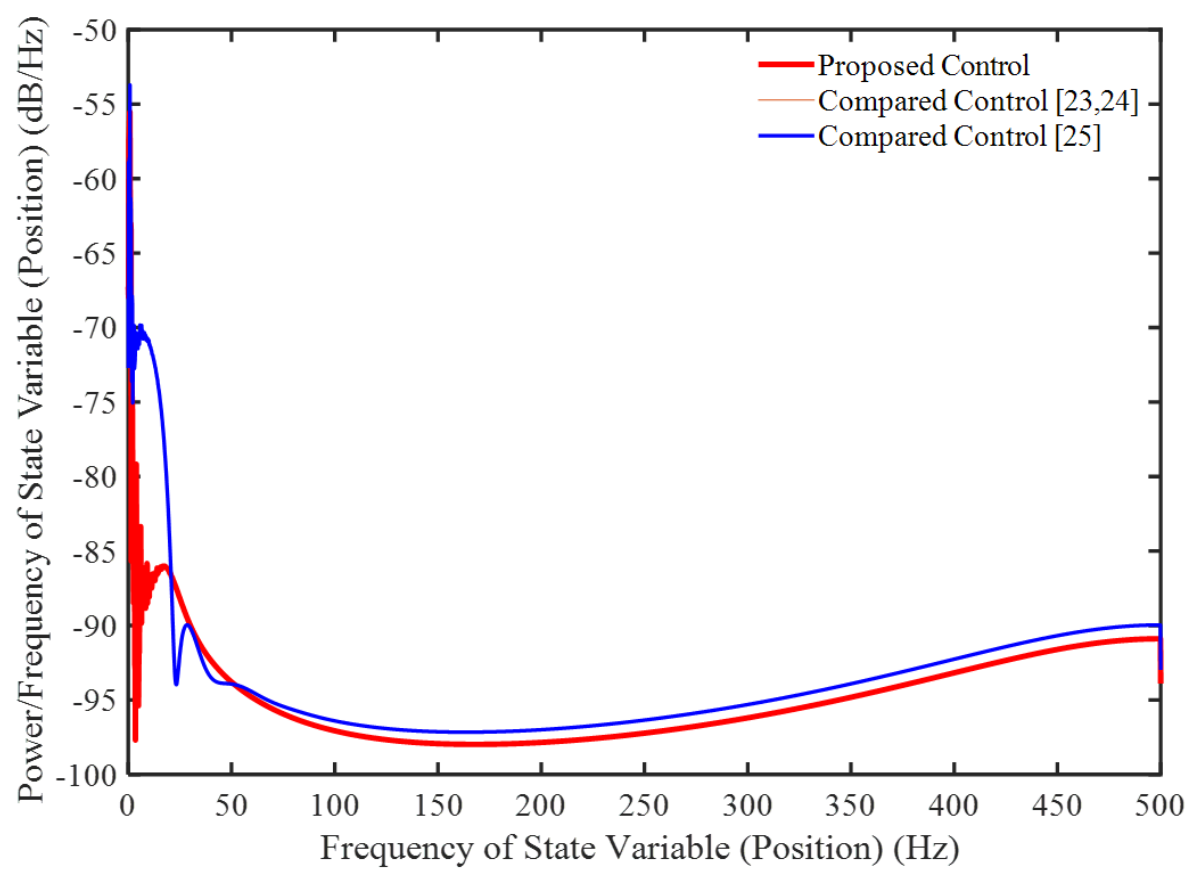

Figure 13. Power spectral density (PSD) of proposed control 1, compared control [23,24], and compared control [25].

\section{Conclusion}

In this study, a new optimal control law was formulated using the sliding mode controller whose gains are adjustable and its control performances were evaluated in terms of power consumption and stability intensity. In the formulation of the controller, the objective function of the Bolza-Meyer criterion was adopted to make the value of the gain larger than one. This is a salient point to resolve some drawbacks of conventional optimal controllers, in which the values of the definite symmetry matrices are less than one. By adopting the adjustability gain values, the non-negative definite symmetric matrix function of the Meyer term is reduced and hence the energy consumption for the control action is also reduced. In order to verify the benefits of the proposed optimal controller, an illustrative example is adopted and its control performance is evaluated based on three criteria: control gain index, main input control index, and the state index. After showing excellent control performance of the proposed controller, it is then applied to vibration control of a vehicle seat suspension system subjected to the random bump road excitation. It is demonstrated that unwanted vibration at the driver position is well controlled by implementing the proposed controller, leading to lower power and higher stability.

It is finally remarked that although the simulation models used in this work have not considered parameter uncertainties or/and disturbances, the proposed optimal control law can be applied straightforwardly to those systems without significant modification. This is possible since the proposed optimal control law has variable-gain values of the sliding mode controller, which is one of the outstanding robust controllers. This 
work will be undertaken in the near future as a second phase of this work. In addition, the proposed control will also be developed with other types of sliding mode control, the fuzzy model/or neural networks, and online control for improve its performance. It will be intentionally applied to other practical engineering system such as suspension and robotics to evaluating its significant properties.

\section{Declaration of conflicting interest}

The authors declare that there is no conflict of interest.

\section{Nomenclature}
$x(t) \in R^{n}$
The state vector of polynomial time-
$u(t) \in R^{l}$ varying system
The control input of polynomial time-
$B_{\xi}(t)$
varying system
$f_{\xi}(x, t)$
The continuous function of time
$f_{\xi}(x, t)$
A polynomial of $n$ variables with time-
$a_{1 \xi}$ dependent continuous coefficients
$a_{1 \xi}$
The vector of dimension $n$
$a_{p \xi}$
The matrix of dimension $n \times n$
The $(p+1)$ dimension tensor of dimension $n \times \ldots(p+1)$ times $\ldots \times n$
$R_{\xi}(t)$
The positive definite symmetric matrix function
$\psi_{\xi}, L_{\xi}(t)$
Symmetric-positive-define matrices
$\alpha$
The positive constant of the Meyer part of the cost function
$Q_{\xi}(t)$
The square symmetric matrix (gain matrix) $(n \times n)$
$H(x, u, q, t)$
The Hamiltonian function

Appendix: Derivation of Eq. (10) 
Consider the optimal control problem for the system (1) with respect to the Bolza criterion without a nonintegral term:

$$
J=\frac{1}{2} \int_{t o}^{T}\left(u^{T}(s) R_{\xi}(s) u(s)+x^{T}(s) L_{\xi}(s) x(s)\right) d s
$$

In this analysis, Eq.(5) is simplified as $q(t)=\alpha Q_{\xi}(t) x(t)$. This simplification is used for objective to find a range of gain values for the system in optimal control. The appearance of $\operatorname{sign}[x(t)]$ will limit the boundary of the gain value, so this expression must be replaced by the original variable $x(t)$.

From the optimal polynomial-quadratic regulator theory [11], the optimal control law is found as follows:

$$
u^{*}(t)=-R_{\xi}^{-1}(t) B_{\xi}{ }^{T}(t)\left(\alpha Q_{\xi}(t) x(t)\right)=-\alpha R_{\xi}^{-1}(t) B_{\xi}{ }^{T}(t) Q_{\xi}(t) x(t)
$$

Where the gain matrix $Q_{\xi}(t)$ is found from $\frac{d q}{d t}=-\frac{\partial H}{\partial x}$, and the Hamilton function $H(x, u, q, t)=\frac{1}{2}\left(u^{T} R_{\xi}(t) u+x^{T} L_{\xi}(t) x\right)+q^{T} \dot{x}(t)$ as follows:

$\alpha \dot{Q}_{\xi}(t) x(t)+\alpha Q_{\xi}(t) \dot{x}(t)=-L_{\xi}(t) x(t)-\left[\frac{\partial f_{\xi}(x, t)}{\partial x}\right]^{T}\left(\alpha Q_{\xi}(t) x(t)\right)$

$\Leftrightarrow \alpha \dot{Q}_{\xi}(t) x(t)=-L_{\xi}(t) x(t)-\left[\frac{\partial f_{\xi}(x, t)}{\partial x}\right]^{T}\left(\alpha Q_{\xi}(t) x(t)\right)+\alpha Q_{\xi}(t) \dot{x}(t)$

$\Leftrightarrow \alpha \dot{Q}_{\xi}(t) x(t)=-L_{\xi}(t) x(t)-\left[\mathrm{a}_{1 \xi}(t)+2 a_{2 \xi}(t) x(t)+3 a_{3 \xi}(t) x(t) x^{T}(t)+\right.$

$+\ldots+p a_{p \xi}(t) \times(\mathrm{t}) \ldots{ }_{p-1} \quad$ times $\left.\ldots x(t)\right]^{T}\left(\alpha Q_{\xi}(t) x(t)\right)$

$-\alpha Q_{\xi}(t)\left(f_{\xi}(x, t)-B_{\xi}(t) R_{\xi}^{-1}(t) B_{\xi}^{T}(t)\left(\alpha Q_{\xi}(t) x(t)\right)\right)$

$\Leftrightarrow \alpha \dot{Q}_{\xi} x(t)=-L_{\xi}(t) x(t)-\left[\mathrm{a}_{1 \xi}(t)+2 a_{2 \xi}(t) x(t)+3 a_{3 \xi}(t) x(t) x^{T}(t)+\right.$

$+\ldots+p a_{p \xi}(t) \mathrm{x}(\mathrm{t}) \ldots{ }_{p-1}$ times $\left.\ldots x(t)\right]^{T}\left(\alpha Q_{\xi}(t) x(t)\right)-$

$-\alpha Q_{\xi}(t)\left[a_{0 \xi}(t)+a_{1 \xi}(t) x(t)+a_{2 \xi}(t) x(t) x^{T}(t)+\ldots a_{p \xi}(t) x(t) \ldots p-1\right.$ times $\left.\ldots x(t)\right]+$

$+\alpha^{2} Q_{\xi}(t) \mathrm{B}_{\xi}(\mathrm{t}) \mathrm{R}_{\xi}^{-1}(t) \mathrm{B}_{\xi}{ }^{T}(t) \mathrm{Q}_{\xi}(\mathrm{t}) \mathrm{x}(\mathrm{t})$

$\Leftrightarrow \dot{Q}_{\xi}(t) x(t)=-\frac{1}{\alpha} L_{\xi}(t) x(t)-\left[\mathrm{a}_{1 \xi}(t)+2 a_{2 \xi}(t) x(t)+3 a_{3 \xi}(t) x(t) x^{T}(t)+\right.$

$+\ldots+p a_{p \xi}(t) \mathrm{x}(\mathrm{t}) \ldots{ }_{p-1}$ times $\left.\ldots x(t)\right]^{T}\left(Q_{\xi}(t) x(t)\right)+$

$-Q_{\xi}(t)\left[a_{0 \xi}(t)+a_{1 \xi}(t) x(t)+a_{2 \xi}(t) x(t) x^{T}(t)+\ldots a_{p \xi}(t) x(t) \ldots p-1\right.$ times $\left.\ldots x(t)\right]+$

$+\alpha Q_{\xi}(t) \mathrm{B}_{\xi}(\mathrm{t}) \mathrm{R}_{\xi}{ }^{-1}(t) \mathrm{B}_{\xi}{ }^{T}(t) \mathrm{Q}_{\xi}(\mathrm{t}) \times(\mathrm{t})$ 


$$
\begin{aligned}
& \Leftrightarrow \dot{Q}_{\xi}(t)=-\frac{1}{\alpha} L_{\xi}(t)-\left[\mathrm{a}_{1 \xi}(t)+2 a_{2 \xi}(t) x(t)+3 a_{3 \xi}(t) x(t) x^{T}(t)+\right. \\
& \left.+\ldots+p a_{p \xi}(t) \mathrm{x}(\mathrm{t}) \ldots p_{p-1} \text { times } \ldots x(t)\right]^{T} Q_{\xi}(t)- \\
& -Q_{\xi}(t) a_{0 \xi}(t) x^{-1}(t)-Q_{\xi}(t)\left[a_{1 \xi}(t)+a_{2 \xi}(t) x(t)+a_{3 \xi}(t) x(t) x^{T}+\cdots+a_{p \xi}(t) x(t) \ldots p_{p-1 \text { times }}, \ldots x(t)\right]+ \\
& +\alpha Q_{\xi}(t) \mathrm{B}_{\xi}(\mathrm{t}) \mathrm{R}_{\xi}{ }^{-1}(t) \mathrm{B}_{\xi}{ }^{T}(t) Q_{\xi}(t)
\end{aligned}
$$

It is noted that the terminal condition $Q_{\xi}(t)=0$, and the optimal control system is defined as:

$$
\dot{x}(t)=f_{\xi}(x, t)-\alpha B_{\xi}(t) R_{\xi}^{-1}(t) B_{\xi}^{T}(t) Q_{\xi}(t) \times(t), x\left(t_{0}\right)=x_{0}
$$

From Eq. (A.2), the new gain matrix $\alpha Q_{\xi}(t) x(t)$ can be rewritten by $Q_{\Delta}=\alpha Q_{\xi}(t)|x(t)|$. The derivative of the new modified gain matrix is derived as follows:

$$
\begin{aligned}
& \dot{Q}_{\Delta}(t)=\frac{d\left(\alpha Q_{\xi}(t) x^{2}(t)\right)}{d t}=\alpha \frac{d Q_{\xi}(t)}{d t}|x(t)|+\alpha Q_{\xi}(t) \frac{d(|x(t)|)}{d t} \\
& =-L_{\xi}(t)|x(t)|-\alpha\left(\left[a_{1 \xi}(t)+2 a_{2 \xi}(t) x(t)+3 a_{3 \xi}(t) x(t) x^{T}+\ldots+p a_{p \xi}(t) x(t) \ldots p-1 \text { times }, \ldots x(t)\right]^{T} Q_{\xi}(t)\right)|x(t)| \\
& -\alpha Q_{\xi}(t) a_{0 \xi}(t)-\alpha\left(Q_{\xi}(t)\left[a_{1 \xi}(t)+a_{2 \xi}(t) x(t)+a_{3 \xi}(t) x(t) x^{T}+\cdots+a_{p \xi}(t) x(t) \ldots_{p-1 \text { times }}, \ldots x(t)\right]|x(t)|\right. \\
& \left.+\alpha^{2} Q_{\xi}(t) B_{\xi}(\mathrm{t}) \mathrm{R}_{\xi}{ }^{-1}(t) B_{\xi}{ }^{T}(t) Q_{\xi}(\mathrm{t})\right)|x(t)|+\alpha \mathrm{Q}_{\xi}(\mathrm{t}) \mathrm{a}_{0 \xi}(t)+\alpha \mathrm{Q}_{\xi}(\mathrm{t})\left[a_{1 \xi}(t)+a_{2 \xi}(t) x(t)+a_{3 \xi}(t) x(t) x^{T}+\cdots\right. \\
& \left.\left.+a_{p \xi}(t) x(t) \ldots{ }_{p-1 \text { times }}, \ldots x(t)\right]|x(t)|-\alpha R_{\xi}^{-1}(t) B_{\xi}{ }^{T}(t) Q_{\xi}(t)|x(t)|\right) \\
& \left.=-L_{\xi}(t)|x(t)|-\alpha\left[a_{1 \xi}(t)+2 a_{2 \xi}(t) x(t)+3 a_{3 \xi}(t) x(t) x^{T}+\ldots+p a_{p \xi}(t) x(t) \ldots p-1 \text { times }, \ldots x(t)\right]^{T} Q_{\xi}(t)\right)|x(t)|
\end{aligned}
$$




\section{References}

[1] Hamidreza Modares, Frank L. Lewis, Mohammad Bagher Naghibi Sistani, Adaptive optimal control constrained input systems using policy iteration and neural networks, IEEE Transactions on Neural Networks and Learning Systems 24(10) (2013) 1513-1525

[2] Wencheng Luo, Yun-Chung Chu, Keck-Voon Ling, Inverse optimal adaptive control for attitude tracking of spacecraft, IEEE Transactions on Automatic Control 50(11) (2005) 1639-1654

[3] Tao Bian, Yu Jiang, Zhong-Ping Jiang, Decentralized adaptive optimal control of large scale systems with application to power systems, IEEE Transactions on Industrial Electronics 62(4) (2015) 2439-2447 [4] Ruizhuo Song, Frank L. Lewis, Qinglai Wei, Huaguang Zhang, Off-policy actor-critic structure for optimal control of unknown systems with disturbances, IEEE Transactions on Cybernetics 46(5) (2016) $1041-1050$

[5] Kyriakos G. Vamvoudakis, Marcio Fantini Miranda, and João P. Hespanha, Asymptotically stable adaptive-optimal control algorithm with saturating actuators and relaxed persistence of excitation, IEEE Transactions on Neural Networks and Learning Systems 27(11) (2016) 2386-2398

[6] Ding Wang, Derong Liu, Qichao Zhang, and Dongbin Zhao, Data-based adaptive critic designs for nonlinear robust optimal control with uncertain dynamics, IEEE Transactions on Systems, Man, and Cybernetics Systems 46(11) (2016) 1544-1555

[7] Manuel Soler, Maryam Kamgarpour, Javier Lloret, and John Lygeros, A hybrid optimal control approach to fuel-efficient aircraft conflict avoidance, IEEE Transactions on Intelligent Transportation Systems 17(7) (2016) 1826-1838

[8] Hamidreza Modares, Frank L. Lewis, Zhong-Ping Jiang, Optimal output-feedback control of unknown continuous-time linear systems using off-policy reinforcement learning, IEEE Transactions on Cybernetics 46(11) (2016) 2401-2410

[9] Michael Basin Dario Calderon-Alvarez, Antonella Ferrara, Sliding mode regulator as solution to optimal control problem, Proceedings of the 47th IEEE Conference on Decision and Control Cancun, Mexico (2008) 2184-2189

[10] Michael Basin Dario Calderon-Alvarez, Optimal controller for uncertain stochastic polynomial systems, Journal of the Franklin Institute 346 (2009) 206-222

[11] Michael Basin Dario Calderon-Alvarez, Sliding mode regulator as solution to optimal control problem for non-linear polynomial systems, Journal of the Franklin Institute 347 (2010) 910-922

[12] Michael Basin, Pablo Rodriguez-Ramirez, Antonella Ferrara, Dario Calderon-Alvarez, Sliding mode optimal control for linear systems, Journal of the Franklin Institute 349 (2012) 1350-1363 
[13] Mehdi Nikkhah and Hashem Ashrafiuon, Kenneth R. Muske, Optimal sliding mode control for underactuated systems, Proceedings of the 2006 American Control Conference Minneapolis, Minnesota, USA (2006) 4688-4693

[14] Do Xuan Phu, Van Mien, Seung Bok Choi, A novel adaptive gain of optimal sliding mode controller for linear time-varying systems, Applied Sciences 9(23) (2019) 5050

[15] Yinyan Zhang, Shuai Li, Xiaoping Liu, Adaptive near-optimal control of uncertain systems with application to underactuated surface vessels, IEEE Transactions on Control Systems Technology 26(4) (2017) 1204-1218

[16] Do Xuan Phu and Seung-Bok Choi, Vibration control of a ship engine system using high-load magnetorheological mounts associated with a new indirect fuzzy sliding mode controller, Smart Mater. Struct. 24 (2015) 025009

[17] Do Xuan Phu, Do Kyun Shin and Seung-Bok Choi, Design of a new adaptive fuzzy controller and its application to vibration control of a vehicle seat installed with an MR damper, Smart Mater. Struct. 24 (2015) 085012

[18] Do Xuan Phu, Ta Duc Huy, Van Mien, Seung Bok Choi, A new composite adaptive controller featuring the neural network and prescribed sliding surface with application to vibration control, Mechanical Systems and Signal Processing 107 (2018) 409-428

[19] G. Ciccarella , M. Dalla Mora and A. Germani, A Luenberger-like observer for nonlinear systems, International Journal of Control 57(3) (1993) 537-556

[20] Yougang Sun, Junqi Xu, Haiyan Qiang, Guobin Lin, Adaptive neural-fuzzy robust position control scheme for maglev train systems with experimental verification, IEEE Trans. on Industrial Electronics 66(11) (2019) 8589-8599

[21] Yougang Sun,Junqi Xu, Haiyan Qiang, Chen Chen, GuoBin Lin, Adaptive sliding mode control of maglev system based on RBF neural network minimum parameter learning method, Measurement 141 (2019) 217-226

[22] You-Gang Sun, Hai-Yan Qiang, Junqi Xu, Da-Shan Dong, The nonlinear dynamics and anti-sway tracking control for offshore container crane on a mobile harbor, Journal of Marine Science and Technology 25(6) (2017) 656-665

[23] Yuqing Chen, Loris Roveda, David J. Braun, Efficiently computable constrained optimal feedback controllers, IEEE Robotics and Automation Letter 4(1) (2019) 121-128

[24] Yuqing Chen, David J. Braun, Hardware-in-the-loop iterative optimal feedback control without model based future prediction, IEEE Transactions on Robotics 35(6) (2019) 1419-1434 
[25] Tianyi Zeng, Xuemei Ren, Yao Zhang, Guang Li, Jing Na, An integrated optimal design for guaranteed cost control of motor driving system with uncertainty, IEEE/ASME Transactions on Mechatronics 24(6) (2019) 2606-2615

[26] Do Kyun Shin, Do Xuan Phu, Sang-Min Choi, Seung-Bok Choi, An adaptive fuzzy sliding mode control of magneto-rheological seat suspension with human body model, Journal of Intelligent Material Systems and Structures 27(7) (2016) 925-934 